\title{
How Lotteries in School Choice Help to Level the Playing Field*
}

\author{
Christian Basteck $^{\dagger} \quad$ Bettina Klaus ${ }^{\ddagger} \quad$ Dorothea Kübler ${ }^{\S}$
}

November 29, 2019

\begin{abstract}
School authorities in the UK and the US advocate the use of lotteries to help desegregate schools. Inspired by the current school choice mechanism in Berlin, we study lottery quotas embedded in the deferred acceptance (DA) and immediate acceptance (IA) mechanisms. Some seats are allocated based on academic achievement (e.g., grades) and some based on a lottery. We focus on the effect of the lottery quota on truth-telling, stability, the utility of students, and the student composition at schools, using theory and experiments. We find that in theory a lottery quota strengthens truth-telling in DA by eliminating non-truth-telling equilibria. The equilibrium outcome of DA with a lottery is stable while this is not the case for IA with a lottery. Both predictions are borne out in the experiment. Moreover, the lottery quota leads to more diverse school populations in the experiment, as predicted. Comparing the two mechanisms, students with the lowest grades profit more from the introduction of the lottery under IA than under DA.
\end{abstract}

Keywords: School choice, immediate acceptance mechanism, deferred acceptance mechanism, lotteries, experiment, market design.

JEL codes: C78, C91, D82, I24.

*The authors would like to thank Bistra Solakova for her contribution to this research in its early phase (see Solakova, 2011) and to Renke Schmacker and Felix Bönisch for excellent research assistance. We are grateful to Nina Bonge who helped us run the experiments and to Jennifer Rontganger for copy editing. We received valuable comments from participants of the Matching-in-Practice workshop in Cologne, the Market Design Workshop at ZEW Mannheim, and the German Society of Operations Research Conference at HU Berlin, the seminar at the MPI for Collective Goods in Cologne, as well as from Bob Hammond. We would like to thank two reviewers, the advisory editor, and the editor for their constructive comments and thoughtful handling of the paper. Bettina Klaus and Dorothea Kübler gratefully acknowledge the hospitality of Stanford University where part of this paper was written. Financial support from Deutsche Forschungsgemeinschaft through CRC TRR 190, from the Swiss National Science Foundation (SNSF) through Project 100018_162606, and from the Fonds de la Recherche Scientifique (FRS-FNRS) through Grant 1.B.222.17F is gratefully acknowledged.

${ }^{\dagger}$ WZB Social Science Center Berlin, Reichpietschufer 50, 10178 Berlin, Germany; e-mail: christian. bastck@wzb.eu.

${ }^{\ddagger}$ Faculty of Business and Economics, University of Lausanne, Internef 538, CH-1015, Lausanne, Switzerland; e-mail: Bettina.Klaus@unil.ch.

${ }^{\S}$ ZZB Social Science Center Berlin, Reichpietschufer 50, 10178 Berlin, Germany, and Technical University of Berlin; e-mail: dorothea.kuebler@wzb.eu. 


\section{Introduction}

School choice has become common practice in urban areas in the US and in Europe. Students are not automatically assigned to a school, but can choose a school that best fulfills their needs and matches their interests. School choice is supposed to permit fair access to good schools and to avoid unjust segregation with respect to socio-demographic characteristics that can arise if there is residential segregation and students have to attend their district school.

However, some of the high hopes in school choice have been dashed by reality. Allowing parents and students to choose among schools has not led to a desegregation of schools, as documented by Allen et al. (2014) for the UK. For Sweden, Bohlmark et al. (2016) even document an increase in segregation following the introduction of school choice procedures. Three distinct reasons have been put forward:

(i) The persistence of segregation could be due to the preferences of students and parents. Homophily may lead parents to choose schools with students of a similar socio-economic background. Also, school segregation can result when there is residential segregation and parents prefer schools in their proximity. ${ }^{1}$ Relatedly, school choice has not always led to a higher demand for schools with a strong academic record than for poorly performing schools. $^{2}$

(ii) School choice mechanisms themselves may inadvertently further segregation. If school choice procedures are complex and it is hard to find the optimal application strategies, then strategically sophisticated, better-informed parents have an advantage when trying to get their children into desirable schools.

(iii) The selection of students based on academic achievements can lead to a segregation of schools along this criterion, i.e., desirable schools admit the high-achieving students. This segregation along the lines of academic achievement may be considered problematic in itself. It is also possible that it leads to segregation according to socio-economic characteristics where they are correlated with academic achievement.

In this paper, we present mechanisms that deal with the second and third reason. In particular, we vary the manipulability and hence the strategic complexity of the admissions procedure. Moreover, we study to what extent a lottery can reduce the segregation of schools along the lines of academic achievement. We take an existing mechanism involving

\footnotetext{
${ }^{1}$ Residential segregation may in turn be reinforced by prioritizing the geographic proximity of applicants which allows affluent parents to gain access to desired schools via the housing market.

${ }^{2}$ For the UK, Allen et al. (2014) write: "Social class differences in school preferences emerge: middle classes tend to value performance and peer group; lower SES [socio-economic status] groups may look for accessibility, friendliness of staff and support for those of lower ability." In a similar vein, school choices in the city of Amsterdam are driven not by the quality of the schools but by their geographical proximity and by the existence of friends who go to the same school, as documented by Ruijs and Oosterbeek (2019).
} 
a lottery, examine it in light of its desegregation properties, and compare it to a natural alternative mechanism.

Segregation along the lines of academic achievement has been at the heart of a longstanding public debate in England. English grammar schools base their admission decisions on students' exam scores. In regions where these selective grammar schools are numerous and attract the highest achieving students, their counterparts, the so-called comprehensive schools, are left with mostly lower achieving students. ${ }^{3}$ To mitigate segregation, the official School Admissions Code 2007 in the UK proposed using lotteries alongside other admission criteria at oversubscribed schools, a proposal supported by Coldron et al. (2008) in their report on secondary school admissions in England. Noden et al. (2014) report that a small but growing number of English schools use lotteries as the main criterion to determine the student priorities. Similarly, in New York City, Educational Option schools use a combination of priorities based on academic performance and a lottery in an attempt to ensure a diverse student composition, but find it increasingly hard to attract high-achieving applicants who instead flock to selective exam schools. ${ }^{4}$

To reduce segregation and equalize educational opportunities, the city of Berlin introduced a new admission procedure in the academic year 2010/2011. Schools are no longer allowed to use geographic proximity as an admission criterion if the number of applicants exceeds the available seats. Instead, a school can assign at most $60 \%$ of the seats based on applicants' academic achievement and has to assign $30 \%$ via a lottery, with the remaining $10 \%$ reserved for cases of hardship. ${ }^{5}$

The introduction of the lottery quota was highly controversial. Left-leaning politicians who favored a less differentiated student composition across schools (and hence a more diverse student composition within schools) called for a larger lottery quota. Steffen Zillich (member of the Berlin parliament for Die Linke, the Left Party) argued that using a lottery opened up highly demanded schools to children from educationally deprived social groups and that lotteries counteract a further differentiation of schools. ${ }^{6}$ Right-leaning politicians criticized a lottery as arbitrary and favored academic achievement as the principal determinant of priorities; according to Mieke Senftleben (member of the Berlin parliament for the FDP, Free Democratic Party) a lottery undermines the principle of merit, as talent and effort become secondary. ${ }^{7}$ A lottery may also weaken incentives for

\footnotetext{
${ }^{3}$ For the prevalence of selective grammar schools across English cities, see Coldron et al. (2008), Table 13 .

${ }^{4}$ Nathanson et al. (2013) find that Educational Option programs are the modal (reported) first preference of low-achieving students while for the remaining students, more than half rank a selective program first and less than one fifth an Educational Option program.

${ }^{5}$ See Basteck et al. (2015) for details on the admission procedure.

${ }^{6}$ Minutes of the plenary session of the Berlin parliament on June 11, 2009.

${ }^{7}$ Minutes of the plenary session of the Berlin parliament on September 24, 2009.
} 
quality improvements by the schools to attract the most able students, as considered, for example, by Hatfield et al. (2016).

Besides the use of a lottery, the Berlin mechanism is controversial in that it applies the immediate acceptance algorithm. This algorithm has been widely used in many cities, notably in Boston where it first attracted the interest of economists. Following protests from parents, and after the involvement of economists who helped design a new mechanism, Boston abandoned the immediate acceptance mechanism in 2005. The main criticism was that under the immediate acceptance mechanism parents had to manipulate their rank-order lists over schools to achieve a good outcome. ${ }^{8}$ Such manipulations required strategic sophistication and information about the demand for the schools. Thus, the mechanism favored strategically sophisticated and better-informed parents over others. ${ }^{9}$ Under the new mechanism in Boston that is based on the deferred acceptance algorithm, parents cannot gain from misrepresenting their true preferences. This property, called strategy-proofness, levels the playing field among parents. Moreover, truthful reports can serve as a valuable feedback to school authorities on the quality of and the demand for particular schools.

In this paper, we use theory and experiments to investigate the existing mechanism in Berlin, and more generally, to understand the influence of a lottery quota on the two most frequently applied matching mechanisms, the immediate and the deferred acceptance mechanism. Following the controversies that accompanied the introduction of a lottery and taking into account the criticism of the immediate acceptance mechanism, we seek to understand whether the mechanism achieves the political goals of a more diverse student composition of schools and hence less segregation along the lines of academic achievement. In addition, we investigate an alternative mechanism with a lottery that is based on the deferred acceptance algorithm. We show how a lottery quota combined with the deferred acceptance mechanism levels the playing field in two of the three dimensions mentioned above: First, it gives students with lower academic achievements a chance to get a seat at their preferred school, thereby reducing segregation according to academic achievement. Second, it reinforces the strategy-proofness of the deferred acceptance mechanism by making it a strict best response for more students to report their true preferences, thereby reducing the complexity of the admission game.

Previous evidence from the field and the laboratory shows that participants in the deferred acceptance mechanism often fail to understand its incentive properties (Chen

\footnotetext{
${ }^{8}$ For this reason, England outlawed the use of immediate acceptance in 2007 (Pathak and Sönmez, 2013).

${ }^{9}$ In support of this often voiced concern, Basteck and Mantovani (2018) find that subjects of lower cognitive ability fare worse than their high ability peers and that they are at a greater disadvantage under the manipulable immediate acceptance than under the strategy-proof deferred acceptance mechanism.
} 
and Sönmez, 2006; Hassidim et al., 2016). Instead, they manipulate their rank-order lists in a systematic fashion (Echenique et al., 2016; Ding and Schotter, 2017; Klijn et al., 2013). A number of recent studies of matching mechanisms are motivated by the question of how to help participants make the right choices (see for example Bó and Hakimov, 2018). Our study contributes to this discussion by showing that a lottery quota in the deferred acceptance mechanism strengthens truth-telling by giving each applicant a small positive probability to get a seat at the most preferred school(s). In particular, this makes misreporting the most preferred school strictly dominated by truth-telling. In equilibrium, a lottery can also make it a strict (rather than a weak) best response to truthfully reveal the complete preferences.

In the context of school choice, lotteries are also employed to break ties. This has been analyzed by Abdulkadiroğlu et al. (2009). In contrast to tie-breaking lotteries, a lottery quota potentially affects everyone, except for students with a very high priority who get a seat at their most preferred school. The uncertainty arising from the lottery about who has a chance at which school strengthens truth-telling.

Another source of uncertainty is incomplete information about other players, e.g., students' preferences. Ehlers and Massó (2015) analyze ordinal Bayes-Nash equilibria for stable mechanisms in two-sided, many-to-one matching markets with incomplete information. ${ }^{10}$ In particular, they show that the set of equilibria shrinks as the support of the common prior expands (Corollary 1b) and that truth-telling is an equilibrium if schools' priorities are perfectly correlated (Proposition 1a). For one-sided many-to-one matching markets (i.e., non-strategic schools) with serial dictatorship, Chen and Pereyra (2018) show that when each student's prior about other students' preferences and school priorities has full support then truth-telling is the unique ordinal Bayes-Nash equilibrium. Featherstone and Niederle (2016) provide an example where truth-telling is the unique ordinal Bayes-Nash equilibrium in anonymous strategies under immediate acceptance with incomplete information about student preferences. In all these papers, uncertainty that is due to incomplete information may strengthen the incentives to tell the truth. In contrast, participants in our setting are uncertain about their lottery number rather than about the preferences of others. Moreover, in our setting schools have two types of seats - those where priority is based on academic achievement, and lottery seats. Nevertheless, we find that, just like uncertainty from incomplete information, the uncertainty introduced by the lottery quota reduces the set of equilibria and strengthens truth-telling incentives under the stable deferred acceptance mechanism. It also tends to increase truth-telling under immediate acceptance in the aggregate, though not for all students.

\footnotetext{
${ }^{10}$ An ordinal Bayes-Nash equilibrium is a Bayes-Nash equilibrium in which each player's equilibrium strategy yields an outcome distribution that first-order stochastically dominates the outcome distribution of all other potential strategies.
} 
The issue of segregation resulting from school choice has been studied in depth by Calsamiglia et al. (2017). They consider a school-choice model with multiple communities and schools as local public goods, thereby enriching the framework of Epple and Romano (1998, 2008) with different school choice mechanisms, namely immediate and deferred acceptance. Families decide where to live, and the quality of a school is affected by the ability of its students. Even in the absence of priorities based on residence or academic achievement, segregation by student ability may arise under immediate acceptance if high ability applicants care more about peer quality than applicants of lower ability. In contrast, deferred acceptance does not give rise to segregation, as long as the ordinal preferences over schools are identical for applicants of different abilities. In our setup preferences over schools are exogenous and peer effects do not affect school quality. Since schools prioritize students based on academic achievement, sorting along this dimension arises under both mechanisms, but is mitigated by the lottery.

We proceed as follows: First, we describe the existing Berlin school choice mechanism and a version of the deferred acceptance mechanism that incorporates a lottery. We show that the deferred acceptance lottery mechanism preserves the desirable properties of the deferred acceptance algorithm, including stability and strategy-proofness. Furthermore, we show that if students' priorities are the same across schools, then adding a lottery to the deferred acceptance mechanism always increases truth-telling in equilibrium (Theorem 1 ). For general priorities, adding a lottery to the deferred acceptance mechanism makes it a strictly dominant strategy to truthfully rank the most preferred school. While adding a lottery to the immediate acceptance mechanism gives some students an incentive to truthfully rank their most preferred school, some students might demote their most preferred school to avoid competition for a lottery seat. Finally, a lottery quota embedded in immediate acceptance leads to unstable outcomes.

To provide an empirical test of the predicted properties of the mechanisms involving lotteries, we conducted an experiment. We consider a setup where preferences over schools are correlated and academic achievement determines the students' priorities at schools. We compare the immediate acceptance mechanism with a lottery quota (which is a stylized version of the mechanism used in Berlin) to the immediate acceptance mechanism without a lottery and to the deferred acceptance mechanism with and without a lottery. This allows us to test whether, on average, the lottery quota leads to more truthful revelation of preferences under both mechanisms, as predicted by theory for the school choice problems we consider. Moreover, we test the predictions on stability by counting the number of blocking pairs. Finally, we consider which students benefit most from the introduction of a lottery in the two mechanisms and what the effects of the lottery are on the distribution of payoffs across students and on the composition of schools. 
The experimental findings support the main theoretical predictions concerning comparisons of the mechanisms. The results show that lotteries increase truth-telling and lead to a more diverse student body at schools with respect to academic achievement. In particular, lotteries harm good-but-not-excellent students who are displaced by students with lower academic achievements but more luck in the lottery. While these findings hold for both school choice mechanisms, the immediate acceptance mechanism leads to more diverse schools than the deferred acceptance mechanism for the same lottery quota, at the cost of being manipulable and less stable. We conclude that where a lottery quota is used to strike a compromise between meritocratic and egalitarian principles, the size of the quota should take into account the mechanism used. At the same time, the fairness aspect of strategy-proofness and stability may be considered.

The paper is organized as follows. Section 2 introduces four school choice mechanisms, namely Immediate Acceptance (IA), Deferred Acceptance (DA), Immediate Acceptance with a lottery quota for one third of the seats (IA33), and Deferred Acceptance with the same lottery quota (DA33). Then, we analyze the effect of a lottery on stability and truth-telling under DA and IA in equilibrium. Section 3 presents the experimental design and the equilibrium analysis for the four mechanisms, including the hypotheses. The experimental results are found in Section 4, and Section 5 concludes.

\section{School Choice Mechanisms}

A school choice problem is a many-to-one matching problem between a set of students and a set of schools that have a limited number of seats to allocate. Each student has strict preferences over schools and each school has strict priorities over students. Schools having priorities as opposed to preferences implies that they do not act strategically, e.g., because priorities are mandated by the local education authorities. Note that in the remainder of the paper, the shorter term "priorities" can be read as "priorities based on academic achievement."

A solution to a school choice problem consists of a matching in which all students are matched to schools or remain unmatched (in our setup all students will be matched to schools in equilibrium). A school choice mechanism is a systematic way of selecting a matching for any given school choice problem, taking schools' priorities and students' (reported) preferences as inputs. The choice of a matching mechanism is a crucial decision that local education authorities need to make. There are several mechanisms that have prevailed in practice, the two most popular being the Immediate Acceptance (aka Boston) mechanism, IA for short, and the Deferred Acceptance (aka Gale-Shapley) mechanism, DA for short. 


\subsection{The Immediate Acceptance Mechanism (IA)}

The immediate acceptance mechanism first requires all students to submit strict preferences over schools and all schools to submit strict priorities over students. Then, IA computes a matching via the following algorithm.

Step 1. Each student applies to the school he ranks first. Each school matches its school seats one by one with the highest priority applicants (it matches all applicants if there are fewer than the number of school seats). All matched students and school seats are removed. All remaining students are rejected and continue to the next step.

Step $k>1$. Each rejected student applies to his best-ranked school that has not yet rejected him, i.e., his $k^{\text {th }}$ ranked school. Each school matches its remaining school seats one by one with the highest priority applicants (it matches all applicants if there are fewer than the remaining number of school seats). All matched students and school seats are removed. All remaining students continue to the next step.

The IA algorithm terminates when all students are matched to school seats (in our setting, students find all schools acceptable and there are enough school seats available).

One of the main shortcomings of IA is that it may give rise to justified envy: a student might prefer another school seat that is assigned to a lower priority student. No justified envy is a key element in the definition of stability: a matching is stable if no student prefers an unassigned school seat to his allotment (non-wastefulness), no student finds his allotment unacceptable (individual rationality), and there is no justified envy (no pairwise blocking). Furthermore, IA is not strategy-proof; that is, a student may obtain a better match by misrepresenting his preferences. Apart from being a strategic robustness property, strategy-proofness in matching models represents a certain notion of fairness. Former Boston Public Schools superintendent Thomas Payzant, in a memo to the Boston School Committee on May 25 2005, described the rationale for switching away from a manipulable school choice mechanism as follows: "A strategy-proof algorithm levels the playing field by diminishing the harm done to parents who do not strategize or do not strategize well." Despite its various shortcomings (see Abdulkadiroğlu and Sönmez, 2003), IA remains popular and is currently being used not only in Berlin, but in many school districts in the US (e.g., Minneapolis, Lee County of Florida, Denver, and Cambridge, Massachusetts) and in other countries (e.g., Spain, see Calsamiglia and Güell, 2018).

\subsection{The Deferred Acceptance Mechanism (DA)}

The deferred acceptance mechanism first requires all students to submit strict preferences over schools and all schools to submit strict priorities over students. Then, DA computes 
a matching via the following algorithm (first proposed by Gale and Shapley, 1962).

Step 1. Each student applies to the school he ranks first. Each school tentatively matches its school seats one by one with the highest priority applicants (it tentatively matches all applicants if there are fewer than the number of school seats). All remaining students are rejected and continue to the next step.

Step $k>1$. Each tentatively matched student applies again to the same school. Each rejected student applies to his best-ranked school that has not rejected him yet. Each school tentatively matches its school seats one by one with the highest priority applicants (it tentatively matches all applicants if there are fewer than the number of school seats). All remaining students are rejected and continue to the next step.

The DA algorithm terminates when all students are tentatively matched to school seats, at which point the current tentative matching becomes final (in our setting, students find all schools acceptable and there are enough school seats available). The resulting matching is stable.

As mentioned before, several authors underline the policy importance of strategyproofness for school choice mechanisms (see, e.g., Abdulkadiroğlu et al., 2006; Pathak and Sönmez, 2008). The argument is not simply that the truthful revelation of preferences is desirable per se, but that strategy-proofness levels the playing field for strategically unsophisticated and less informed students by making the simple strategy of reporting preferences truthfully weakly dominant. In fact, DA's strategy-proofness was one of the main reasons why the Boston and New York City public school systems, in collaboration with a team of economists (see, e.g., Abdulkadiroğlu, Pathak, and Roth, 2005; Abdulkadiroğlu, Pathak, Roth, and Sönmez, 2005; Pathak and Sönmez, 2008), decided to switch from an IA-based mechanism that was in place to a DA-based mechanism.

\subsection{The Immediate Acceptance Mechanism with a Lottery (IA33)}

In Berlin, the school year 2010/2011 saw the introduction of a new admission procedure for secondary schools. Geographical priorities were abolished. Instead, $60 \%$ of seats are allocated based on academic achievement and another $30 \%$ by lottery. ${ }^{11}$ We model the new Berlin procedure as an IA mechanism in which one third $(33 \%)$ of the seats are matched using a (single) lottery. ${ }^{12}$ Note that the lottery quota is filled after allocating

\footnotetext{
${ }^{11}$ The remaining $10 \%$ are reserved for cases of hardship. See Basteck et al. (2015) for details on the admission procedure.

${ }^{12}$ In practice, Berlin uses multiple school-specific lotteries. However, they are only used in the first step of the mechanism. Hence, a student participates in at most one lottery, so that running a single lottery is
} 
seats based on academic achievement. Following the arguments in Dur et al. (2018), this increases the impact of the lottery by improving the chances of students with worse grades to obtain seats at better schools. (If seats dedicated to students with good grades were allocated after lottery seats then some students with very good grades could occupy lottery seats - hence, fewer students with worse grades might profit from the lottery.)

The immediate acceptance mechanism with a 33\% lottery first requires all students to submit strict preferences over schools and all schools to submit strict priorities over students. Next, a lottery ranking of students is drawn randomly (from a uniform distribution). For simplicity, we assume that the number of seats at each school is a multiple of three. Then, based on the lottery ranking of students, IA33 computes a matching via the following algorithm.

Step 1. Each student applies to the school he ranks first. Each school matches the first two thirds of its school seats one by one with the highest priority applicants and the remaining one third of its school seats one by one with the applicants with the best lottery numbers (it matches all applicants if there are fewer than the number of school seats). All matched students and school seats are removed. All remaining students are rejected and continue to the next step.

Step $k>1$. Each rejected student applies to his best-ranked school that has not rejected him yet, i.e., his $k^{\text {th }}$ ranked school. Each school matches its remaining school seats one by one with the highest priority applicants (it matches all applicants if there are fewer than the remaining number of school seats). All matched students and school seats are removed. All remaining students continue to the next step.

The IA33 algorithm terminates when all students are matched to school seats (in our setting, students find all schools acceptable and there are enough school seats available).

The IA33 outcome is a probabilistic matching, i.e., a convex combination over all deterministic matchings obtained via the above algorithm where each of the underlying lottery rankings occurs with equal probability. For any given realization of the lottery, the IA33 mechanism corresponds to a choice-based immediate acceptance mechanism as introduced and characterized in Doğan and Klaus (2018). Note that, just like IA, IA33 is not strategy-proof.

equivalent to running multiple lotteries. 


\subsection{The Deferred Acceptance Mechanism with a Lottery (DA33)}

The purpose of introducing a lottery in the Berlin public school system was to give students with lower academic achievements a shot at being placed at popular schools with positive probability and to limit the concentration of these students at unpopular schools. ${ }^{13}$ It is well understood, especially since the redesign of the Boston and New York city public school assignment, that strategy-proofness and stability are key properties of a well-functioning school choice mechanism. In this section, we introduce a lottery-based variant of the DA mechanism in which one third (33\%) of school seats are matched using a lottery. As in Berlin and under IA33, in each step the lottery quota is filled after allocating the seats based on academic achievement, which increases the impact of the lottery on the final matching.

The deferred acceptance mechanism with a 33\% lottery first requires all students to submit strict preferences over schools and all schools to submit strict priorities over students. Next, a lottery ranking of students is drawn randomly (from a uniform distribution). ${ }^{14}$ For simplicity, we assume that each school has a number of school seats that is a multiple of three. Then, based on the lottery ranking of students, DA33 computes a matching via the following algorithm.

Step 1. Each student applies to the school he ranks first. Each school tentatively matches the first two thirds of its school seats one by one with the highest priority applicants and the remaining one third of its school seats one by one with the applicants with the best lottery numbers (it tentatively matches all applicants if there are fewer than the number of school seats). All remaining students are rejected and continue to the next step.

Step $k>1$. Each tentatively matched student applies again to the same school. Each rejected student applies to his best-ranked school that has not rejected him yet. Each school tentatively matches the first two thirds of its school seats one by one with the highest priority applicants and the remaining one third of its school seats one by one with the applicants with the best lottery numbers (it tentatively matches all applicants if there are fewer than the number of school seats). All remaining students are rejected and continue to the next step.

The DA33 algorithm terminates when all students are tentatively matched to school seats. Then, the current tentative matching becomes final (in our setting, students find all schools acceptable and there are enough school seats available).

\footnotetext{
${ }^{13}$ See Basteck et al. (2015) for details on the political objectives.

${ }^{14}$ In line with our modeling of IA33, we use a single lottery rather than school-specific lotteries.
} 
The DA33 outcome is a probabilistic matching, i.e., a convex combination over all deterministic matchings obtained via the above algorithm where each of the underlying lottery rankings occurs with equal probability. For any given realization of the lottery, the DA33 mechanism corresponds to a choice-based deferred acceptance mechanism as introduced and characterized in Ehlers and Klaus (2016). Note that, just as for DA, DA33 is strategy-proof, due to the tentative assignment of both priority and lottery seats.

\subsection{The Effect of a Lottery on Stability and Truth-Telling in Equilibrium}

In this section we consider preference revelation games under complete information, generated by the four mechanisms. Students evaluate distributions of assignment probabilities based either on expected utility or on stochastic dominance. That is, a student strictly prefers a probability distribution over another if and only if the former yields a higher expected utility or, alternatively, if and only if the former stochastically dominates the latter. ${ }^{15}$ A profile of preference reports by the students is a (pure strategy Nash) equilibrium in a school choice game (under a mechanism) if no student can achieve a strictly preferred probability distribution over schools by changing his preference report.

We next define ex post stability for our two lottery mechanisms: a mechanism with a lottery quota is ex post stable if for each realization of the lottery no student prefers an unassigned school seat to his allotment (non-wastefulness), no student finds his allotment unacceptable (individual rationality), and there is no justified envy (no pairwise blocking) defined as follows: we divide the school seats into priority (66\%) and lottery (33\%) seats; then a student prefers neither a priority seat that is assigned to a lower priority student nor a lottery seat that is assigned to a student with a lower lottery number. ${ }^{16}$

Remark 1 (Ex Post Stability in Equilibrium). Under DA, DA33, and IA all equilibrium outcomes are (ex post) stable (with respect to true preferences). For IA this follows from Ergin and Sönmez (2006) while for DA and DA33 it follows from the fact that, given that assignments are tentative, it is a dominant strategy to report all desirable schools a student might obtain truthfully (including those that might be obtained only via the lottery). Equilibrium outcomes of the IA33 mechanism can be ex post unstable due to justified envy: since applicants do not know their lottery number, it can be ex

\footnotetext{
${ }^{15}$ In our school choice model, probability distribution $\mathbb{P}_{1}$ stochastically dominates probability distribution $\mathbb{P}_{2}$ if the following holds: the probability to be matched to the most preferred school under $\mathbb{P}_{1}$ is at least as high as under $\mathbb{P}_{2}$, the probability to be matched to the two most preferred schools under $\mathbb{P}_{1}$ is at least as high as under $\mathbb{P}_{2}$, the probability to be matched to the three most preferred schools under $\mathbb{P}_{1}$ is at least as high as under $\mathbb{P}_{2}$, etc., with one of these probabilities being strictly higher for $\mathbb{P}_{1}$.

${ }^{16}$ See Westkamp (2013) for a related definition of stability.
} 
ante optimal for an applicant to skip his preferred school in equilibrium. If it turns out later that this applicant's lottery number was very good, he might ex post envy another applicant's lottery seat at a preferred school.

Recall that DA is strategy-proof and hence truthful reporting is a weakly dominant strategy. There may, however, exist non-truth-telling equilibria, for example, when applicants demote schools in their ranking at which they stand no chance of being admitted. There is ample empirical evidence of such skipping behavior, often leading to the safe (district) school being ranked first. Skipping is also observed in contexts where students do not know how good their chances at the schools are. ${ }^{17}$ Introducing a lottery not only gives every applying student a non-zero chance of being admitted, but also makes this clear to students. Hence, we have the following:

Fact 1 (For General Priorities, in Equilibrium under DA33, First-Ranked Schools Must be Reported First). Since truth-telling is weakly dominant and since a lottery ensures that students are admitted to their first-ranked school whenever they are ranked first by the lottery, it is a strictly dominated strategy (dominated by truth-telling) not to rank the most preferred school first. In particular, in all equilibria of the school choice game under DA33, students truthfully reveal their most preferred school.

Furthermore, by eliminating some of the non-truth-telling equilibria, the introduction of a lottery can lead to more truth-telling in equilibrium. For the case where student priorities are the same across schools, for example, when they are based on exam scores or other measures of academic achievement, we are able to state this formally in Theorem 1 below. ${ }^{18}$

\section{Theorem 1 (For Common Priorities, an Equilibrium under DA33 is an Equi- librium under DA but not Vice Versa).}

Assume students' priorities are the same across schools and assume either that students compare probability distributions over schools based on expected utility or that they compare them by stochastic dominance. Then,

(a) any equilibrium in the school choice game under DA33 is an equilibrium in the school choice game under DA, and

(b) some equilibria in the school choice game under DA are not equilibria in the school choice game under DA33 (and hence violate truth-telling in ways that cannot arise in an equilibrium under DA33).

\footnotetext{
${ }^{17}$ Experimental studies that document skipping the top choice or reversals under DA are Chen and Sönmez (2006), Pais and Pinter (2008), Ding and Schotter (2017), Klijn et al. (2013), and in a two-sided matching context Echenique et al. (2016). This literature is surveyed by Hakimov and Kübler (2019).

${ }^{18}$ We state the result for DA33, but it holds more generally for any positive lottery quota.
} 
Theorem 1 implies that if students' priorities are the same across schools then the set of equilibria in the school choice game under DA33 is a strict subset of the set of equilibria under DA. Since both sets of equilibria include truth-telling as an equilibrium, all of the additional equilibria that occur under DA involve some students not telling the truth; in this sense there is relatively more truth-telling in equilibrium under DA33 than under DA.

Proof of Theorem 1. (a) Let $P$ be a profile of students' preference reports over schools that is an equilibrium in the school choice game under DA33. We show that $P$ is also an equilibrium in the school choice game under DA.

Assume, toward a contradiction, that $P$ is not an equilibrium in the school choice game under DA. Then, at profile $P$, some student $i$ can get a preferred school by reporting different preferences. Since truth-telling is a weakly dominant strategy under DA, student $i$ can also get a preferred school by reporting his true preferences $P_{i}^{t}$.

Now, recall that DA33 produces a probability distribution by using the deterministic DA33 algorithm for each possible lottery ranking and by taking the average over the obtained outcomes. Furthermore, in the deterministic DA33 algorithm for any lottery ranking, it is a weakly dominant strategy for student $i$ to report his true preferences $P_{i}^{t}$. Hence, for any lottery ranking, student $i$, when telling the truth $P_{i}^{t}$ instead of $P_{i}$, will get the same or a (strictly) preferred school. Moreover, for the particular lottery ranking that coincides with the priorities of schools over students, the associated deterministic DA33 and DA coincide. Thus, with our previous observation that student $i$ is assigned a (strictly) preferred school under DA when he reports the truth $P_{i}^{t}$ instead of $P_{i}$, we can conclude that the probability distribution of DA33 when student $i$ tells the truth $P_{i}^{t}$ stochastically dominates and hence yields a strictly higher expected utility than the probability distribution of DA33 based on $P_{i}$. This is a contradiction to $P$ being an equilibrium in the school choice game under DA33.

(b) The following example shows that the set of equilibria in the school choice game under DA33 can be a strict subset of the set of equilibria in the school choice game under DA.

We assume that there are two schools, $A$ and $B$, each with three seats and a common priority ranking over students $1,2, \ldots, 6$ such that students are labeled by their rank. All students consider $A$ to be a better school than $B$. Under DA, students 1,2 , and 3 are assigned to $A$ and students 4,5 , and 6 are assigned to $B$. It is an equilibrium in the school choice game under DA that students 1, 2, and 3 truthfully report school $A$ as their best school while students 4,5 , and 6 falsely report school $B$ as their best school. This is not an equilibrium in the school choice game under DA33 because each of the students 4 , 5 , and 6 has a positive probability of being assigned to school $A$ if he ranks it first and hence would have an incentive to unilaterally deviate to truth-telling. 
Note that the restriction to common priorities in Theorem 1 cannot be dispensed with: if the schools' priorities over students are sufficiently heterogeneous, for instance, as when they are given by walkzones, then the introduction of a lottery can create new non-truth-telling equilibria. The following example illustrates this.

Example 1 (For General Priorities, an Equilibrium under DA33 might not be an Equilibrium under DA). Consider three schools, $A, B$, and $C$, with three seats each, one at each school reserved for the lottery quota, and a common priority ranking over students $1,2, \ldots, 6$ such that students are labeled by their rank. Both schools $A$ and $B$ rank students $1,2, \ldots, 6$ higher than the remaining three students 7,8 , and 9 . Schools' priorities and students' preferences are as follows (entries marked with $\diamond$ are not relevant).

\begin{tabular}{l||c|c|c|c|c|c||c|c|c} 
schools' priorities & 1st & 2nd & 3 rd & 4 th & 5 th & 6 th & 7 th & 8 th & 9 th \\
\hline \hline$A$ & 1 & 2 & 3 & 4 & 5 & 6 & 7 & 8 & 9 \\
\hline$B$ & 1 & 2 & 3 & 4 & 5 & 6 & 9 & 7 & 8 \\
\hline$C$ & $\diamond$ & $\diamond$ & $\diamond$ & $\diamond$ & $\diamond$ & $\diamond$ & $\diamond$ & $\diamond$ & $\diamond$
\end{tabular}

\begin{tabular}{|c|c|c|c|}
\hline students' preferences & 1 st & $2 \mathrm{nd}$ & $3 r d$ \\
\hline $1 \& 2$ & $A$ & $\diamond$ & $\diamond$ \\
\hline $3 \& 4$ & $B$ & $\diamond$ & $\diamond$ \\
\hline $5 \& 6$ & $C$ & $\diamond$ & $\diamond$ \\
\hline 7 & $B$ & $A$ & $C$ \\
\hline $8 \quad \& \quad 9$ & $A$ & $B$ & $C$ \\
\hline
\end{tabular}

In the truth-telling equilibrium under DA33, students 1 and 2 report school $A$ as their best and are admitted (based on their rank), while students 3 and 4 as well as 5 and 6 are likewise admitted to schools $B$ and $C$. Students 7, 8, and 9 compete for the lottery seats at $A$ and $B$ (one per school). It is easy to see that given any lottery ranking, student 7 will never be assigned to school $A$ : if student 7 is first in the lottery ranking, he will be assigned to school $B$ while if either student 8 or 9 are first in the lottery ranking, one of them will be assigned to school $A$. Hence, a unilateral deviation from truth-telling by student 7 where he demotes $A$ and reports $B$ as his best and $C$ as his second best school yields the same outcome and is still an equilibrium under DA33. 
We show that the above non-truth-telling equilibrium under DA33 is not an equilibrium under DA. Under truth-telling, students 1-6 are admitted to the same school as before. For student 7 , we now find that he is admitted to $A$ (his second-most preferred school) while student 9 is admitted to $B$. If student 7 was to deviate unilaterally by demoting $A$ in his reported ranking - reporting $B$ as his best and $C$ as his second best school - he would still not be admitted to $B$ but would instead be admitted to $C$, making him strictly worse off than under truth-telling.

Finally, consider the effect of a lottery on truth-telling under immediate acceptance. We rely on the following classification of students.

Fact 2. In any equilibrium of the school choice game under IA, we can classify students as those who

(i) are admitted to their most preferred school.

(ii) see their $k$ most preferred schools filled by applicants of higher priority and have to rank their $(k+1)^{\text {th }}$ most preferred school first in order to be admitted there.

(iii) see their $k$ most preferred schools filled by applicants of higher priority and are admitted to their $(k+1)^{\text {th }}$ most preferred school even without ranking it first.

For students of type (i) it is a best response to rank their most preferred school first while students of type (ii) have a strict incentive to misreport their preferences. Students of type (iii) are indifferent between several (mis)reports, including ranking any of their $(k+1)$ most preferred schools first. Introducing a lottery gives applicants of type (ii) and (iii) a positive probability of obtaining their most preferred school if they rank it first, and hence tends to increase their incentives to report truthfully. However, some students of type (i) can lose their safe priority seat as we move from IA to IA33 and may hence misreport their most preferred school, opting for a safe seat at a less competitive school instead of competing for a lottery seat. Thus, while the introduction of a lottery increases the incentives to report preferences truthfully for some students, its overall effect on truth-telling is ambiguous.

\section{Design of the Experiment}

The experiment is the first to investigate behavior under the two most important school choice mechanisms, IA and DA, in combination with a lottery quota. We study the effect of a lottery on truth-telling, stability, the utility of students, and the student composition at schools. 


\subsection{The Experimental School Choice Problems}

Each participant in the experiment is part of a group of 12 students who are competing for seats at three schools. The three schools differ in size and popularity. Schools $A$ and $B$ have three seats each while school $C$ has six seats. School $A$ is the most popular school in that it is most preferred by nine of the 12 students. School $B$ is the second-most popular school in that it is most preferred by three out of 12 students. School $C$ is the unanimously least preferred school and all students prefer any school over being unmatched. Thus, our setting features two preference types - a majority of students whose preferences are $A \succ B \succ C \succ \emptyset$ (A-types) and a minority whose preferences are $B \succ A \succ C \succ \emptyset$ (B-types) - and aims to capture both a high correlation of preferences ( $A$ is the most and $C$ the least preferred school) as well as some degree of heterogeneity.

Schools have the same strict priorities over students, which we interpret as a ranking of students by academic achievement. To simplify notation, we identify students' names and their relative position in this ranking: student 1 is the strongest student enjoying the highest priority, student 2 the second strongest, etc., and student 12 is the weakest student.

Table 1 indicates how we construct the 20 different school choice problems, namely the preference profiles for the 12 students identified by their rank $i$ (the complete profiles can be found in Appendix D.1, Table 12).

Table 1: Preference Profiles

\begin{tabular}{l||c|c|c|c}
$i$ & $P 1$ & $P 2$ & $\ldots$ & $P 20$ \\
\hline \hline $1-6$ & $A$ & $A$ & $A$ & $A$ \\
7 & $A$ & $A$ & $\ldots$ & $B$ \\
8 & $A$ & $A$ & $\ldots$ & $B$ \\
9 & $A$ & $B$ & $\ldots$ & $B$ \\
10 & $B$ & $A$ & $\ldots$ & $A$ \\
11 & $B$ & $B$ & $\ldots$ & $A$ \\
12 & $B$ & $B$ & $\ldots$ & $A$ \\
\hline
\end{tabular}

Notes: Preference types (most preferred schools) of students 1-12 in profiles $P 1-P 20$.

We assume that the six strongest students 1-6 prefer school $A$, while the three students who prefer $B$ are ranked between 7 and $12 .{ }^{19}$ Permuting the rank of the three students

\footnotetext{
${ }^{19}$ We therefore focus on cases in which there is a positive correlation between academic rank and a
} 
who most prefer $B$, we arrive at 20 different preference profiles, and hence 20 different experimental school choice problems used in our treatments. We will denote the set of $A$-type students in rank $7-12$ by $7-12(A)$ and the set of $B$-type students in rank $7-12$ by $7-12(B)$.

To induce the preference profiles in the experiment, students receive a payoff that depends on both the school he is matched to and his own type. $A$-type students receive $22 €$ if admitted to $A$ and $16 €$ if admitted to $B$ while the payoffs for $B$-type students are reversed - they receive $22 €$ if admitted to $B$ and $16 €$ if admitted to $A$. All students receive $10 €$ if admitted to $C$ and $0 €$ if not admitted to any school. While it is possible for a student to be unassigned if he lists too few schools, he can always guarantee a school seat for himself by ranking enough schools, so that school $C$ is effectively the safety school in our experiment.

\subsection{Equilibrium Predictions}

We analyze the corresponding school choice games for our mechanisms (IA, IA33, DA, and DA33) assuming that students maximize their expected payoffs.

Equilibria under IA. In every equilibrium of the school choice game under IA and any of the preference profiles $P 1-P 20$ :

- Students 1-3 rank A first (not ranking A first is strictly dominated) and are admitted.

- Students 4-6 rank B first (strict best response) and are admitted.

- Students 7-12 are admitted to $C$ (they can report any ranking that includes $C$ ).

\begin{tabular}{l||c|c|c|c} 
students & $1-3$ & $4-6$ & $7-12(A)$ & $7-12(B)$ \\
\hline \hline match probability at $A$ & 1 & 0 & 0 & 0 \\
\hline match probability at $B$ & 0 & 1 & 0 & 0 \\
\hline match probability at $C$ & 0 & 0 & 1 & 1
\end{tabular}

In equilibrium, at least three students truthfully report their most preferred school (students 1-3) and at least three students (students 4-6) misrepresent their preferences. All six remaining students (students 7-12) are always matched to school $C$ as long as they include it in their rankings. We derive the set of equilibria in detail in Appendix A.1.

preference for the top school. The converse case where stronger applicants prefer the less popular school would yield a trivial matching outcome. 
Equilibria under IA33. In every equilibrium of the school choice game under IA33 and any of the preference profiles P1-P20:

- Students 1-2 rank A first (not ranking A first is strictly dominated) and are admitted.

- Students 3-4 rank B first (strict best response) and are admitted.

- All other A-type students with ranks between 5-12 rank $A$ first and include $C$ in their ranking (strict best response). They are admitted to $A$ with probability $1 / 5=0.2$ and are otherwise admitted to $C$.

- All three B-type students with ranks between 7-12 rank $B$ first and include $C$ in their ranking (strict best response). They are admitted to $B$ with probability $1 / 3 \approx 0.333$ and are otherwise admitted to $C$.

\begin{tabular}{l||c|c|c|c} 
students & $1-2$ & $3-4$ & $5-12(A)$ & $7-12(B)$ \\
\hline \hline match probability at $A$ & 1 & 0 & 0.2 & 0 \\
\hline match probability at $B$ & 0 & 1 & 0 & 0.333 \\
\hline match probability at $C$ & 0 & 0 & 0.8 & 0.667
\end{tabular}

In equilibrium, apart from students 3 and 4 , all students truthfully report their most preferred school. Intuitively, the two top students (students 1-2) can always obtain their most preferred school $A$. The two next best students (students $3-4$ ) would have to enter a lottery for the one remaining seat at school $A$, where they have no priority over lower-ranked applicants, or they can apply to school $B$ and be sure to be matched to their second-most preferred school; in equilibrium, they opt for the latter. With both top schools almost filled, lower-ranked students (students 5-12) have to decide at which school they want to enter the lottery for the remaining seat. This choice is governed by their type. We derive the set of equilibria in detail in Appendix A.2.

Equilibria under DA. In every equilibrium of the school choice game under DA and any of the preference profiles $P 1-P 20$ :

- Students 1-3 rank A first (not ranking A first is strictly dominated) and are admitted.

- Students 4-6 rank B first, or second behind $A$, and are admitted to B.

- Students 7-12 are admitted to $C$ (they can report any ranking that includes $C$ ). 


\begin{tabular}{l||c|c|c|c} 
students & $1-3$ & $4-6$ & $7-12(A)$ & $7-12(B)$ \\
\hline \hline match probability at $A$ & 1 & 0 & 0 & 0 \\
\hline match probability at $B$ & 0 & 1 & 0 & 0 \\
\hline match probability at $C$ & 0 & 0 & 1 & 1
\end{tabular}

In equilibrium, at least three students truthfully report their most preferred school (students 1-3). For all students it is a weakly dominant strategy to report their true preferences, but three students (4-6) may misreport $B$ to be most preferred and the remaining students (7-12) will be matched to $C$ as long as they include it in their rankings. We derive the set of equilibria in detail in Appendix A.3.

Equilibria under DA33. In every equilibrium of the school choice game under DA33 and any of the preference profiles $P 1-P 20$ :

- Students 1-2 rank A first (not ranking A first is strictly dominated) and are admitted.

- Students 3-12 report their true preferences (not ranking the most preferred school first is strictly dominated and truthfully ranking all schools is a strict best response).

- Students 3-4 are admitted to $A$ with probability $2 / 15 \approx 0.133$ and are otherwise admitted to $B$.

- Student 5 is admitted to $A$ with probability $2 / 15 \approx 0.133$, to $B$ with probability $34 / 105 \approx 0.324$, and is otherwise admitted to $C$.

- All four A-type students with ranks between 6-12 are admitted to $A$ with probability $2 / 15 \approx 0.133$, to $B$ with probability $3 / 35 \approx 0.086$, and are otherwise admitted to $C$.

- All three B-type students with ranks between 7-12 are admitted to $A$ with probability $1 / 45 \approx 0.022$, to $B$ with probability $1 / 5=0.2$, and are otherwise admitted to $C$.

\begin{tabular}{l||c|c|c|c|c} 
students & $1-2$ & $3-4$ & 5 & $6-12(A)$ & $7-12(B)$ \\
\hline \hline match probability at $A$ & 1 & 0.133 & 0.133 & 0.133 & 0.022 \\
\hline match probability at $B$ & 0 & 0.867 & 0.324 & 0.086 & 0.2 \\
\hline match probability at $C$ & 0 & 0 & 0.543 & 0.781 & 0.778
\end{tabular}


In contrast to DA, the lottery ensures that in the DA33 mechanism every student has a chance of being admitted to his first-ranked school. Hence, all students rank their most preferred school first and all but students 1 and 2 have an incentive to truthfully report their complete preferences. We derive the set of equilibria in detail in Appendix A.4.

Remark 2 (IA and DA have the same Equilibrium Outcome). Since there is a unique stable matching, the results by Ergin and Sönmez (2006, Theorem 1) imply that the unique equilibrium outcomes for our experimental school choice problems under IA and DA are the same (although the underlying strategy profiles can differ). This feature of our markets greatly simplifies the comparison of the results of the two mechanisms with a lottery.

Remark 3 (Equilibria for Arbitrary Lottery Quotas). Appendix B presents our school choice problem in a continuum model, allowing us to vary the lottery quotas continuously and to derive the corresponding equilibria under both mechanisms for arbitrary quotas. Besides presenting a robustness check on whether the comparative statics between mechanisms continue to hold for lottery quotas other than one third and zero, the model also allows for robustness checks with respect to various preference distributions and school sizes (see Appendix B for details). We mention these findings in the context of Hypotheses 5 and 6 .

\subsection{Experimental Design and Procedures}

\section{Treatments}

We employ a between-subjects design where each treatment is devoted to one of the four mechanisms (IA, IA33, DA, and DA33). Treatment IA33 is based on the mechanism that is used in Berlin for secondary schools. The 20 school choice problems described in Table 1 were used in every treatment in the order of their presentation in Appendix D.1. For each school choice problem, the students' ranks were randomly assigned anew to the participants, and each participant received a new lottery ranking in treatments IA33 and DA33. Thus, each participant played 20 school choice games with changing roles and with slightly different preference profiles. While students were aware of their priority ranking based on academic achievement when submitting their preferences over schools, the lottery ranking was not disclosed to them until the end of the round. This reflects the fact that students have a good idea of their grade point averages when applying to secondary schools, but that the lottery ranking is drawn only after the applications have been submitted. 


\section{Experimental Procedures}

The computerized experiment was conducted at the TU-WZB Experimental Lab in Berlin. ${ }^{20}$ Overall, we conducted 16 sessions, with four sessions for each of the treatments IA, IA33, DA, and DA33, respectively. There were 24 subjects in a session, divided into two matching groups of 12 participants. Subjects stayed together in this group of 12 for the entire 20 rounds of the experiment. Thus, we end up with eight independent observations and data from $4 \times 24=96$ participants per treatment, yielding a total number of 384 subjects. The sessions lasted an average of 90 minutes and subjects earned around 20€. The experiment was programmed with z-Tree (Fischbacher, 2007). Each subject participated in only one session.

In each session, subjects were randomly assigned to the computer terminals in the lab. They were given written instructions. To ensure that everybody understood the tasks, we conducted a quiz before starting with the experiment. For the quiz, the subjects had to work through examples of allocating seats in a school choice problem. They had to apply the same algorithm as the one used in their respective treatment. We checked their written answers and clarified all remaining questions in private.

An English translation of the experimental instructions and the quiz as well as screenshots (in German) can be found in Appendix D.

\subsection{Hypotheses}

\section{Truth-Telling in Equilibrium}

Table 2 shows which students are predicted to truthfully reveal their full preferences, truthfully report their most preferred school, etc. As we move from IA to DA or from IA33 to DA33, truth-telling becomes a (weakly) dominant strategy and hence we expect truth-telling rates to increase. Moving from IA to DA, this effect should be strongest for the good-but-not-excellent students who are not good enough to ensure themselves a seat at their most preferred school, but would, in equilibrium, be good enough to be admitted to their second-most preferred school under immediate acceptance, provided they misreport their preferences and rank it first. This yields our first hypothesis.

Hypothesis 1. On average, truth-telling increases as we move from IA to DA and from IA33 to DA33.

\footnotetext{
${ }^{20}$ The first sessions were run in 2011 and formed part of a master's thesis (Solakova, 2011). Additional sessions were run in 2015 and 2017. All sessions were conducted by the lab manager Nina Bonge in the same lab with the same subject pool. The 2011 sessions provide us with half of the data set for treatments DA, IA, and IA33 (48 participants each). They yield results very similar to the sessions conducted later with the same treatments.
} 
Table 2: Preference Reports in Equilibrium

\begin{tabular}{l||c|c|c|c}
$i$ & IA & DA & IA33 & DA33 \\
\hline \hline 1 & $\bar{t}$ & $\overline{\boldsymbol{t}}$ & $\overline{\boldsymbol{t}}$ & $\overline{\boldsymbol{t}}$ \\
2 & $\overline{\boldsymbol{t}}$ & $\overline{\boldsymbol{t}}$ & $\overline{\boldsymbol{t}}$ & $\overline{\boldsymbol{t}}$ \\
3 & $\overline{\boldsymbol{t}}$ & $\overline{\boldsymbol{t}}$ & $\boldsymbol{\ell}$ & $\boldsymbol{t}$ \\
4 & $\boldsymbol{\ell}$ & $\underline{\boldsymbol{t}}$ & $\boldsymbol{\ell}$ & $\boldsymbol{t}$ \\
5 & $\underline{\boldsymbol{t}}$ & $\underline{\boldsymbol{t}}$ & $\boldsymbol{t}$ \\
6 & $\boldsymbol{\ell}$ & $\overline{\boldsymbol{t}}$ & $\boldsymbol{t}$ \\
$7-12(A)$ & $\boldsymbol{c}$ & $\boldsymbol{c}$ & $\overline{\boldsymbol{t}}$ & $\boldsymbol{t}$ \\
$7-12(B)$ & $\boldsymbol{c}$ & $\boldsymbol{c}$ & $\overline{\boldsymbol{t}}$ & $\boldsymbol{t}$ \\
\hline
\end{tabular}

Notation:

$\boldsymbol{t}$ indicates that applicants reveal their full preferences truthfully, $\bar{t}$ indicates that applicants rank their most preferred school first, $\underline{t}$ indicates that applicants rank their least preferred below their secondmost preferred school,

$\ell$ indicates that applicants misrepresent their most preferred school and rank their second-most preferred school first, and

$\boldsymbol{c}$ indicates that applicants include school $\mathrm{C}$ in their ranking. 
Next, we consider the effect of a lottery on truth-telling under immediate acceptance. Moving from IA to IA33 increases the chances for students 5-12 to be admitted to their most preferred school when ranking it first. Hence, we expect truth-telling to increase for these students. ${ }^{21}$ The overall increase in truth-telling is tempered by the fact that student 3 is no longer guaranteed a seat at school $A$ and may refrain from truth-telling to avoid the lottery at school $A .^{22}$

Hypothesis 2. On average, truth-telling increases as we move from IA to IA33.

For deferred acceptance, truth-telling is an equilibrium under both DA and DA33 but the introduction of the lottery eliminates most of the non-truth-telling equilibria that exist besides truth-telling under DA (see Theorem 1 and Table 2). The lottery quota makes it a dominated strategy to not report the most preferred school truthfully and makes reporting the true preferences over all three schools a strict best response for all but the two top-ranked students. Without the lottery, truth-telling is never a strict best response in equilibrium. Truthfully revealing the most preferred school is a strict best response only for the three best students in the equilibrium under DA, and truthfully ranking the second-most over the least preferred school $C$ is a strict best response only for students $4-6$.

Hypothesis 3. On average, truth-telling increases as we move from DA to DA33.

\section{Stability, Payoff Distribution, and Student Composition of Schools}

Stability is a desirable property of matching outcomes since it guarantees that no student can claim that he should get a seat at a more preferred school, without violating the priorities of other students. For the mechanisms with a lottery, we use a notion of ex post stability where we divide the school seats into those allocated according to priority based on academic achievement (66\%) and according to the lottery (33\%). Ex post stability requires that a student neither prefers a priority seat that is assigned to a student with worse grades nor prefers a lottery seat that is assigned to a student with a worse lottery number. In our experiment, all assignments are individually rational. Hence, we only need to count student-school blocking pairs (with either a priority or a lottery violation) and non-wastefulness violations (students who did not receive a vacant school seat).

In equilibrium, only IA33 leads to ex post unstable outcomes in our experimental school choice problems. The reason for the instability of IA33 is the following: Under IA33, a student has to decide whether to rank a school first and therefore possibly participate

\footnotetext{
${ }^{21}$ See Section 2.5, Fact 2 and the subsequent discussion of type-(ii) and type-(iii) students.

${ }^{22}$ See Section 2.5, Fact 2 and subsequent discussion of type-(i) students.
} 
in its lottery. Suppose that in equilibrium, it is optimal for a student to skip his most preferred school (e.g., student 3, see Section 3.2). However, if this student has a good lottery number, he can form a blocking pair with his preferred school $A$ if it admitted a student with a worse lottery number for the lottery seat. In DA33, this is impossible since lottery seats are only tentatively assigned in every step.

Hypothesis 4. The outcome under DA, IA, and DA33 is stable while the outcome under IA33 can be unstable, that is, blocking pairs can exist.

The main political objective of introducing a lottery in Berlin was to equalize access to schools for students of varying academic achievement. We therefore investigate whether the lottery leads to a more equitable distribution of payoffs among students and to smaller differences in the student compositions across schools. Moreover, we analyze whether the lottery is more effective in reaching these goals when used in combination with IA or DA.

In theory, the lottery quota reduces the importance of academic rank. Our analysis of a continuum model in Appendix B.3 demonstrates that this holds for arbitrary lottery quotas and under a wider range of preference profiles and school sizes than those of our experimental markets: a higher lottery quota reduces the mass of admissions based on rank and increases the chances of the lower-ranked applicants who rely on the lottery. For our experimental markets, Table 3 shows the expected payoffs in equilibrium for students of each rank across treatments. Students 3,5, and 6 lose when the lottery is introduced under IA and DA while the lower-ranked students $7-12$ gain. ${ }^{23}$ Given that in general the lower-ranked students expect lower payoffs in equilibrium, the redistribution of payoffs toward these students reduces the inequality in expected payoffs as measured by the Gini index, presented in the last row of Table 3.

Next, we consider the effect of the lottery on the student composition of schools. Table 4 displays the expected average ranks of students at the schools under the four mechanisms. Providing lower-ranked students with access to more popular schools through a lottery reduces differences in the student composition between schools.

The following hypothesis summarizes the effects of introducing a lottery quota for IA and DA.

Hypothesis 5. Moving from IA to IA33 and from DA to DA33,

(i) increases the average payoffs of the lower-ranked students, but decreases the average payoffs of the good-but-not-excellent students,

\footnotetext{
${ }^{23}$ No reduction in payoff is predicted for student 4 because in equilibrium his rank still ensures him a seat at school $B$ under both IA33 and DA33.
} 
Table 3: Expected Equilibrium Payoffs

\begin{tabular}{l||c|c|c}
$i$ & IA/DA & IA33 & DA33 \\
\hline \hline 1 & 22.00 & 22.00 & 22.00 \\
2 & 22.00 & 22.00 & 22.00 \\
3 & 22.00 & 16.00 & 16.80 \\
4 & 16.00 & 16.00 & 16.80 \\
5 & 16.00 & 12.40 & 13.54 \\
6 & 16.00 & 12.40 & 12.11 \\
$7-12(A)$ & 10.00 & 12.40 & 12.11 \\
$7-12(B)$ & 10.00 & 14.00 & 12.53 \\
\hline Sum of payoffs & 174.00 & 180.00 & 177.20 \\
Average payoff & 14.50 & 15.00 & 14.77 \\
Gini index & 0.181 & 0.111 & 0.121 \\
\hline
\end{tabular}

Notes: Expected equilibrium payoffs for students 1-12 in each treatment. Students 7-12 may be of preference type $A$ or $B$.

Table 4: Expected Average Student Rank at Schools

\begin{tabular}{l||c|c|c}
$i$ & IA/DA & IA33 & DA33 \\
\hline \hline$A$ & 2 & 3.63 & 3.27 \\
$B$ & 5 & 5.5 & 5.45 \\
$C$ & 9.5 & 8.43 & 8.64 \\
$\Delta_{A B}$ & 3 & 1.87 & 2.18 \\
$\Delta_{B C}$ & 4.5 & 2.93 & 3.19 \\
$\Delta_{A C}$ & 7.5 & 4.8 & 5.37 \\
\hline
\end{tabular}

Notes: Averages of the equilibrium outcomes for all 20 preference profiles $P 1-P 20 . \Delta_{i j}$ denotes the difference in average ranks between schools $i$ and $j$. 
(ii) leads to a more equitable distribution of expected payoffs across ranks as measured by the Gini index; and

(iii) decreases the difference between the average ranks of students at the three schools.

Finally, we compare the two mechanisms with a lottery, DA33 and IA33. Notice that good-but-not-excellent students (i.e., those who are just below the cutoff to be admitted to the most competitive school $A$ based on their academic rank) have an advantage under DA33 compared to IA33. Under DA33, they can first enter a lottery at their most preferred school and, if they are rejected, they can still apply to their second-most preferred school where they are likely to be admitted based on their relatively high rank. In contrast, under IA33 these students have to decide whether to apply for the lottery seat at $A$ or whether to rely on their relatively high rank and apply to $B$ where they are most likely admitted in Step 1. The fact that these good-but-not-excellent students fare better under DA33 than under IA33 comes at the expense of lower-ranked students who are less likely to be admitted to their most preferred school. As a result, IA33 redistributes more strongly toward lower-ranked applicants than DA33. For a theoretical analysis of this effect in our experimental markets, see Section 3.2. Our analysis of a continuum model in Appendix B shows that the same effect holds for arbitrary lottery quotas and more general preference profiles and school sizes (see Proposition 1 and Corollary 1).

Moreover, by benefiting the lower-ranked students, IA33 also has a stronger equalizing effect with respect to school composition than DA33. Theoretical predictions for our experimental markets are displayed in Table 4. Appendix B, Proposition 2, shows that the comparative statics extend to arbitrary quotas and to more general preference profiles and school sizes.

Hypothesis 6. Comparing IA33 to DA33,

(i) the good-but-not-excellent students receive higher average payoffs under DA33 while the lower-ranked students fare better under IA33;

(ii) inequality, as measured by the Gini index, is lower under IA33 than under DA33; and

(iii) schools are more similar with respect to the average rank of their students under IA33 than under DA33.

We note in passing that we expect no differences in the distribution of payoffs and the composition of schools when comparing IA and DA without a lottery, since the matching outcomes are identical in equilibrium (see Remark 2). 


\section{Experimental Results}

We first analyze the observed preference manipulations across the four mechanisms (IA, IA33, DA, and DA33). Then, we investigate the payoffs of students, the distributional effects of the lottery quota, and the composition of schools.

\section{Truth-Telling and Equilibrium Choices}

We say that a participant is telling the truth if he states his full preferences truthfully. ${ }^{24}$ Figure 1 reports the average truth-telling rates across mechanisms.

Figure 1: Average Truth-Telling Rates

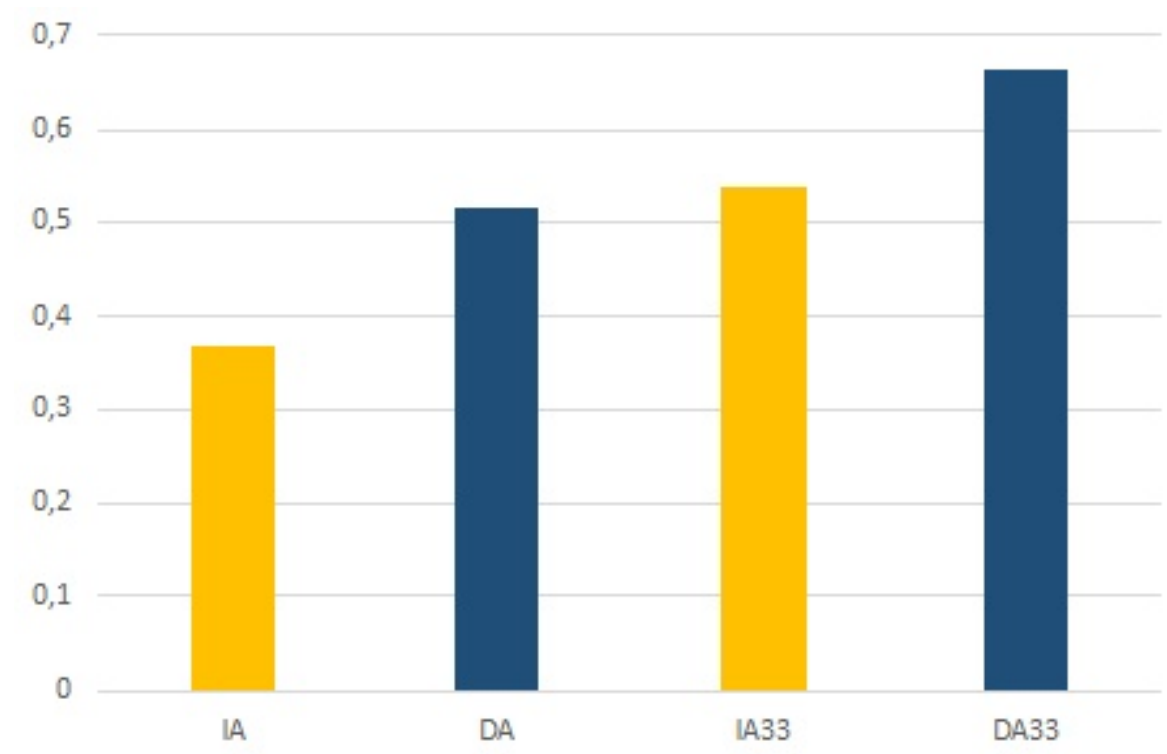

Notes: The figure displays the proportion of participants per treatment that submit their full rankings truthfully.

The left panel of Table 5 presents the average truth-telling rates at each rank separately and the average across ranks. The test results can be found in the right panel. In line with Hypothesis 1, we find that, on average, truth-telling rates are significantly higher under DA than under IA, as indicated by the MWU test with $p=.002$ in the last row of the right panel. Moreover, the effect is strongest for the good-but-not-excellent students $4-6$, significant at $p=.001$. Again, in support of Hypothesis 1, average truth-telling rates are significantly higher under DA33 than IA33 $(p=.005)$. We find the strongest increase in truth-telling for students 3 and 4 and a smaller significant increase for students 2 and 5. Hence, as hypothesized, the increase in truth-telling due to a switch from immediate to deferred acceptance is most pronounced for good-but-not-excellent students.

\footnotetext{
${ }^{24}$ The results are qualitatively unchanged if we consider truthful reports of the most preferred school only (the corresponding analysis is available upon request from the authors).
} 
Table 5: Observed Truth-Telling Rates

\begin{tabular}{|c|c|c|c|c|c|c|c|c|}
\hline \multirow[b]{2}{*}{$i$} & \multicolumn{4}{|c|}{$\begin{array}{l}\text { Truth-Telling Rates } \\
\text { (treatment averages) }\end{array}$} & \multicolumn{4}{|c|}{$\begin{array}{c}\text { Mann-Whitney U Test } \\
\text { ( } p \text {-values })\end{array}$} \\
\hline & IA & $\mathrm{DA}$ & IA33 & DA33 & $\begin{array}{l}\text { IA vs. } \\
\text { DA }\end{array}$ & $\begin{array}{l}\text { IA33 vs. } \\
\text { DA33 }\end{array}$ & $\begin{array}{l}\text { IA vs. } \\
\text { IA } 33\end{array}$ & $\begin{array}{l}\text { DA vs. } \\
\text { DA33 }\end{array}$ \\
\hline 1 & .756 & .794 & .813 & .85 & .561 & .363 & .492 & .262 \\
\hline 2 & .8 & .813 & .781 & .894 & .705 & .017 & .706 & .071 \\
\hline 3 & .794 & .8 & .288 & .631 & .872 & .001 & .001 & .014 \\
\hline 4 & .15 & .519 & .219 & .581 & .001 & .001 & .096 & .397 \\
\hline 5 & .081 & .45 & .406 & .669 & .001 & .008 & .001 & .006 \\
\hline 6 & .069 & .444 & .431 & .594 & .001 & .071 & .001 & .038 \\
\hline $7-12(A)$ & .162 & .377 & .567 & .644 & .003 & .225 & .001 & .002 \\
\hline $7-12(B)$ & .429 & .406 & .615 & .608 & .489 & .958 & .002 & .001 \\
\hline $1-12$ & .369 & .514 & .54 & .665 & .002 & .005 & .002 & .005 \\
\hline
\end{tabular}

Notes: The left panel presents the average truth-telling rates, i.e., the proportion of subjects submitting the full ranking of three schools truthfully. In the right panel, the Mann-Whitney $\mathrm{U}$ test is based on eight matching group averages per treatment. All $p$-values with $p \leq 0.05$ are in bold.

Result 1. The average truth-telling rate increases as we move from IA to DA and from IA33 to DA33.

Next, consider the effect of a lottery quota on truth-telling. Moving from IA to IA33 increases overall truth-telling, as predicted. In particular, students $4-6$ (for student 4 significant only at the $10 \%$ level) as well as students ranked 7-12 truthfully reveal their preferences more often. Note that these are precisely the students for which the lottery increases the chances of being admitted to their most preferred school if they rank it first. At the same time, the introduction of the lottery induces student 3 to misrepresent his preferences significantly more often, since he loses his priority seat at school A. These observations are in line with Hypothesis 2.

Result 2. The average truth-telling rate increases as we move from IA to IA33.

Finally, we consider the effect of a lottery on truth-telling under DA. Moving from DA to DA33, we find that the truth-telling rates increase significantly for students ranked 
5 and below. We observe that only student 3, who loses his priority seat at his most preferred school under DA33, misrepresents his preferences more often under DA33 than under DA, in contrast to the theoretical prediction. Hence, in line with Hypothesis 3 we find:

Result 3. The average truth-telling rate increases as we move from DA to DA33.

Note that the rate of truth-telling is statistically indistinguishable for DA and IA33 (MWU test, $p=0.293$ ). Thus, if higher rates of truth-telling are a political objective (e.g., to allow school authorities to learn more about true preferences) but changing the mechanism is difficult, adding a lottery to IA can be an option.

We can also compare the proportions of equilibrium choices across the four mechanisms, based on the predictions in Table 2. Table 6 displays that subjects choose the equilibrium strategies in IA and DA most of the time. Thus, many non-truth-telling strategies under DA are inconsequential, as most students manage to rank their most preferred school first (students 1-3) or their least preferred below their second preferred school (students 4-6). However, equilibrium choices are less frequent with a lottery quota (IA33 and DA33) for all except the two best students. For example, students 5 and 6 in IA33 follow the equilibrium strategy to rank school A first less than $50 \%$ of the time. The equilibrium strategy in DA33 requires that all three schools have to be submitted truthfully by students 3-12. We find that, on average, $62 \%$ of choices are in line with this.

Table 6: Proportions of Equilibrium Strategies

\begin{tabular}{l||r|r|r|l}
$i$ & IA & DA & IA33 & DA33 \\
\hline \hline 1 & 0.975 & 0.994 & 1.000 & 0.981 \\
2 & 0.981 & 0.988 & 0.969 & 0.969 \\
3 & 0.969 & 0.956 & 0.688 & 0.631 \\
4 & 0.838 & 0.881 & 0.756 & 0.581 \\
5 & 0.906 & 0.881 & 0.438 & 0.669 \\
6 & 0.913 & 0.881 & 0.475 & 0.594 \\
$7-12(A)$ & 0.998 & 0.994 & 0.625 & 0.644 \\
$7-12(B)$ & 0.998 & 0.998 & 0.771 & 0.608 \\
\hline
\end{tabular}

Notes: The table displays the proportions of decisions that are in line with the equilibrium strategies stated in Table 2. 


\section{Stability, Payoff Distribution, and Student Composition of Schools}

We now turn to the experimental results on matching outcomes, and first consider stability. Figure 2 displays the number of blocking pairs in the four treatments. In line with the predictions, the number of blocking pairs is highest in IA33. However, we also observe unstable outcomes in the other three mechanisms. They are due to out-of-equilibrium choices of the participants. The number of blocking pairs is lowest in DA, and almost identical in IA and DA33.

Figure 2: Number of Blocking Pairs

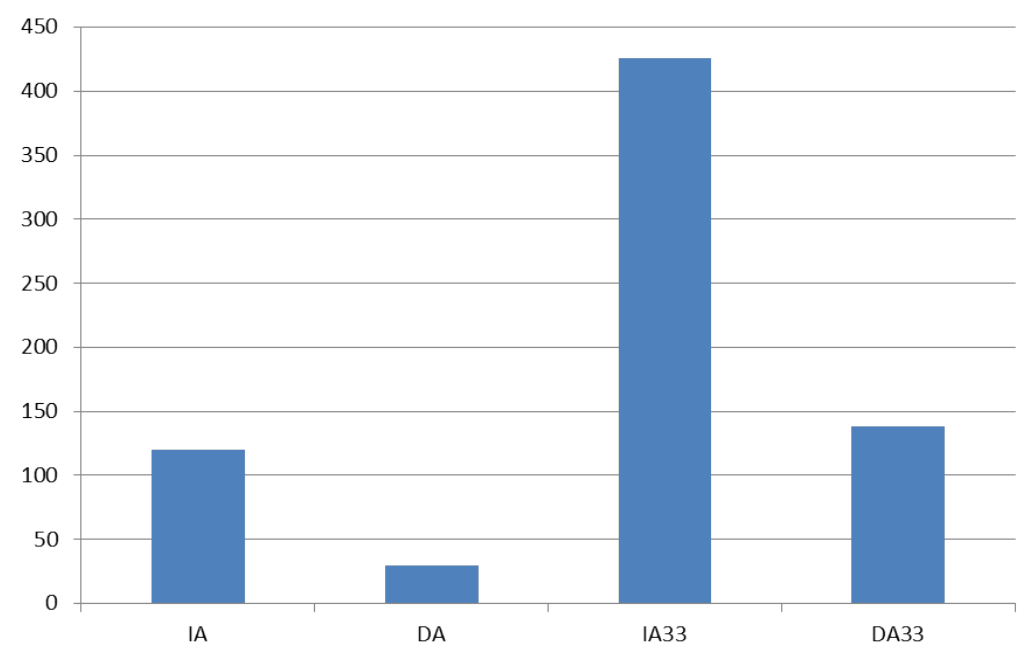

Notes: The figure displays the total number of blocking pairs by treatment.

Applying MWU tests to the number of blocking pairs per matching group over 20 rounds yields no significant difference between IA and DA $(p=.143)$. We find that lotteries significantly increase the number of blocking pairs $(p=.011$ for DA versus DA33 and $p=.000$ for IA versus IA33). Furthermore, as predicted DA33 is more stable than IA33 $(p=.001) \cdot{ }^{25}$

Result 4. The number of blocking pairs is lowest in DA, followed by IA. Both mechanisms are less stable with a lottery quota. As predicted, IA33 displays the highest number of blocking pairs.

We next consider the students' payoffs. It turns out that almost all students were matched to a school. Out of 1,920 observations per treatment (eight groups of 12 students

\footnotetext{
${ }^{25} \mathrm{We}$ can also study the source of the instability, that is, whether the blocking pair is due to the priority based on grades or the lottery, see Table 10 in Appendix C. Moreover, Table 11 in Appendix C shows at which ranks the blocking pairs occur.
} 
over 20 rounds), a student remained unmatched in four cases in IA $(0.2 \%)$, in 10 cases in IA33 $(0.5 \%)$, in one case in DA $(0.05 \%)$, and in three cases in DA33 $(0.15 \%)$.

Table 7: Individual Payoffs

\begin{tabular}{|c|c|c|c|c|c|c|c|c|}
\hline \multirow[b]{2}{*}{$i$} & \multicolumn{4}{|c|}{$\begin{array}{c}\text { Payoffs } \\
\text { (treatment averages) }\end{array}$} & \multicolumn{4}{|c|}{$\begin{array}{c}\text { Mann-Whitney U Test } \\
\text { ( } p \text {-values })\end{array}$} \\
\hline & IA & DA & IA33 & DA33 & $\begin{array}{l}\text { IA vs. } \\
\text { DA }\end{array}$ & $\begin{array}{c}\text { IA33 vs. } \\
\text { DA33 }\end{array}$ & $\begin{array}{l}\text { IA vs. } \\
\text { IA33 }\end{array}$ & $\begin{array}{l}\text { DA vs. } \\
\text { DA33 }\end{array}$ \\
\hline 1 & 21.738 & 21.925 & 22.000 & 21.850 & .441 & .144 & .144 & .538 \\
\hline 2 & 21.888 & 21.888 & 21.713 & 21.775 & .644 & .952 & .522 & .334 \\
\hline 3 & 21.775 & 21.700 & 14.550 & 16.863 & .637 & .001 & .001 & .001 \\
\hline 4 & 15.113 & 16.075 & 15.363 & 16.750 & .003 & .001 & .336 & .001 \\
\hline 5 & 15.513 & 15.963 & 13.763 & 13.525 & .026 & .672 & .002 & .001 \\
\hline 6 & 15.413 & 15.963 & 12.675 & 12.525 & .012 & .916 & .001 & .001 \\
\hline $7-12(A)$ & 10.480 & 10.004 & 12.592 & 11.804 & .001 & .005 & .001 & .001 \\
\hline $7-12(B)$ & 10.492 & 10.275 & 12.871 & 12.154 & .239 & .010 & .001 & .001 \\
\hline $1-12$ & 14.529 & 14.529 & 14.704 & 14.597 & .915 & .052 & .010 & .064 \\
\hline Gini index & .174 & .179 & .116 & .136 & .114 & .001 & .001 & .001 \\
\hline
\end{tabular}

Notes: The left panel displays the average realized payoffs and the Gini index. Averages are taken for the eight matching groups where for each group we compute the average payoff at a given rank over the 20 periods. In the right panel, the Mann-Whitney $\mathrm{U}$ test is based on the eight matching group averages per treatment. All $p$-values with $p \leq 0.05$ in bold.

The left panel of Table 7 displays the average payoffs for each student rank. Note that the lowest feasible average payoff for the group of 12 participants is 13, while the highest is $16 .{ }^{26}$ In the right panel, we compare the average payoffs per rank between treatments using MWU tests.

Our school choice problems were designed such that the unique equilibrium outcome under DA is equal to the unique equilibrium outcome under IA. Hence, there should be no differences between the mechanisms with respect to average equilibrium payoffs without a lottery quota. However, the individual payoffs of students 4-6 and 7-12(A) differ under

\footnotetext{
${ }^{26}$ In the worst case, school $A$ is filled with $B$-types and school $B$ with $A$-types, while in the best case, both schools $A$ and $B$ are filled only with students of their respective type.
} 
these two mechanisms. This is due to out-of-equilibrium behavior that harms students 4-6 and gives students 7-12 an advantage under IA relative to DA.

Introducing a lottery under IA and DA redistributes the payoffs between the students as predicted. For both mechanisms, the lottery significantly reduces the payoffs of students 3,5 , and 6 while it increases the payoffs of lower-ranked students which is reflected in the Gini coefficient. Student 4 is sure to get (at least) a seat at $B$ in equilibrium both with and without the lottery. He experiences a small significant payoff increase in DA33 relative to DA.

As an ordinal measure of welfare, we consider the probability of receiving the favorite school. It is displayed in Table 8 which is constructed in the same way as Table $7 .{ }^{27}$

Table 8: Probability of Receiving the Favorite School

\begin{tabular}{|c|c|c|c|c|c|c|c|c|}
\hline \multirow[b]{2}{*}{$i$} & \multicolumn{4}{|c|}{$\begin{array}{c}\text { Probability } \\
\text { (treatment averages) }\end{array}$} & \multicolumn{4}{|c|}{$\begin{array}{c}\text { Mann-Whitney U Test } \\
\text { ( } p \text {-values })\end{array}$} \\
\hline & IA & $\mathrm{DA}$ & IA33 & DA33 & $\begin{array}{l}\text { IA vs. } \\
\text { DA }\end{array}$ & $\begin{array}{l}\text { IA33 vs. } \\
\text { DA33 }\end{array}$ & $\begin{array}{l}\text { IA vs. } \\
\text { IA } 33\end{array}$ & $\begin{array}{l}\text { DA vs. } \\
\text { DA33 }\end{array}$ \\
\hline 1 & .975 & .994 & 1.00 & .981 & .440 & .144 & .143 & .488 \\
\hline 2 & .981 & .988 & .969 & .969 & .589 & 1.00 & .563 & .298 \\
\hline 3 & .969 & .956 & .056 & .150 & .596 & .018 & .001 & .001 \\
\hline 4 & .013 & .038 & .069 & .125 & .223 & .027 & .009 & .003 \\
\hline 5 & .062 & .019 & .156 & .138 & .264 & .705 & .001 & .001 \\
\hline 6 & .019 & .006 & .138 & .138 & .488 & .831 & .002 & .009 \\
\hline $7-12 \mathrm{~A}$ & .006 & .000 & .156 & .096 & .063 & .015 & .001 & .000 \\
\hline $7-12 B$ & .040 & .023 & .219 & .146 & .236 & .005 & .001 & .001 \\
\hline $1-12$ & .258 & .256 & .293 & .269 & .332 & .007 & .001 & .038 \\
\hline
\end{tabular}

Notes: The left panel displays the probability of receiving a seat at the most preferred school (treatment averages per rank). The right panel displays the MWU test results for the probability of ending up at the most preferred school. All $p$-values with $p \leq 0.05$ in bold.

Overall, this measure leads to results similar to the welfare analysis based on payoffs. The lottery quota redistributes the probability of getting admitted to one's favorite school

\footnotetext{
${ }^{27} \mathrm{We}$ can also consider the probability of receiving the least preferred school $C$. This leads to qualitatively similar results.
} 
from students who lose their priority by grade (student 3 in our markets) to all other students with worse grades. Table 8 also shows that there is no significant difference between IA and DA, as predicted, while IA33 compared to DA33 benefits students 7-12 to the detriment of students 3 and 4 .

Beyond its effect on individual payoffs, we also find that the introduction of a lottery leads to a more equal student composition across schools with respect to average ranks. Figure 3 and Table 9 present the average student ranks at the three schools.

Figure 3: Composition of Schools

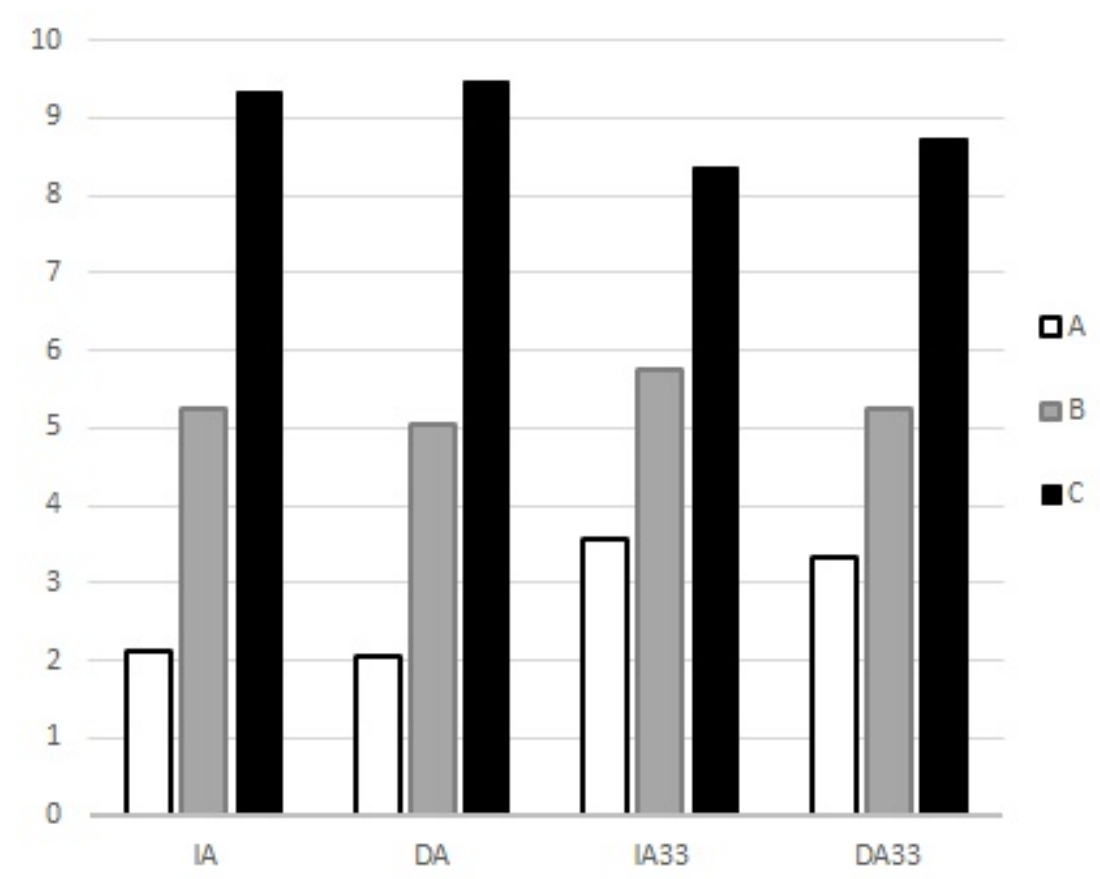

Notes: The bars indicate the average rank of students assigned to each school.

The difference in average ranks between schools $A$ and $C$ is larger under IA and DA than under the two mechanisms with a lottery, IA33 and DA33. Moreover, the same is true for the difference between $A$ and $B$ as well as $B$ and $C$ (all significant at $p=.001$ ). Thus, lotteries have the desired effect of making schools more similar in terms of admitted students' academic achievement, both under IA and under DA. This is in line with Hypothesis 5.

Result 5. Moving from IA to IA33 and from DA to DA33

(i) makes the lower-ranked students better off in terms of average payoffs and the probability of obtaining their preferred school while making the good-but-not-excellent students worse off, 
Table 9: Composition of Schools

\begin{tabular}{c||cccc||cccc}
\multicolumn{1}{l||}{} & \multicolumn{3}{c||}{$\begin{array}{c}\text { Average Student Rank } \\
\text { (treatment averages) }\end{array}$} & \multicolumn{5}{c}{$\begin{array}{c}\text { Mann-Whitney U Test } \\
(p \text {-values })\end{array}$} \\
& IA & DA & IA33 & DA33 & IA vs. & IA33 vs. & IA vs. & DA vs. \\
& & & & & DA & DA33 & IA33 & DA33 \\
\hline \hline \multirow{1}{*}{$A$} & 2.11 & 2.04 & 3.57 & 3.31 & & & & \\
$B$ & 5.25 & 5.04 & 5.76 & 5.25 & & & & \\
$C$ & 9.33 & 9.46 & 8.36 & 8.72 & & & & \\
$\Delta_{A B}$ & 3.14 & 3.00 & 2.19 & 1.94 & $\mathbf{0 . 0 1 5}$ & 0.142 & $\mathbf{0 . 0 0 1}$ & $\mathbf{0 . 0 0 1}$ \\
$\Delta_{B C}$ & 4.01 & 4.42 & 2.60 & 3.47 & $\mathbf{0 . 0 0 3}$ & $\mathbf{0 . 0 0 1}$ & $\mathbf{0 . 0 0 1}$ & $\mathbf{0 . 0 0 1}$ \\
$\Delta_{A C}$ & 7.21 & 7.42 & 4.79 & 5.41 & $\mathbf{0 . 0 2 7}$ & $\mathbf{0 . 0 0 2}$ & $\mathbf{0 . 0 0 1}$ & $\mathbf{0 . 0 0 1}$ \\
\hline
\end{tabular}

Notes: $\Delta_{i j}$ denotes the difference in average ranks between schools $i$ and $j$. The Mann-Whitney $\mathrm{U}$ tests are based on the 8 matching group averages per treatment. All $p$-values with $p \leq 0.05$ are in bold.

(ii) leads to a more equitable distribution of average payoffs across students, as measured by the Gini index, and

(iii) decreases the difference between the average ranks of students at the three schools.

With respect to Hypothesis 6, we compare the distribution of payoffs and the composition of schools between IA33 and DA33. The data confirm the prediction that the beneficial effect of a lottery for lower-ranked students is more pronounced under IA33 than under DA33. Students 7-12 earn significantly more under IA33, both A-types $(p=.005)$ and B-types $(p=.010)$, see Table 7 . On the other hand, students 3 and 4 are better off under DA33. Also note that IA33 leads to higher average payoffs than DA33 (marginally significant at $p=.052$ ). This is in line with the theoretical results of Abdulkadiroğlu et al. (2011) and Miralles (2009) regarding the higher ex ante efficiency of IA compared to DA in the presence of tie-breaking lotteries. ${ }^{28}$ The same comparisons hold when we consider an ordinal measure of welfare (see Table 8).

The Gini-index is significantly lower for IA33 than for DA33. Moreover, IA33 leads to

\footnotetext{
${ }^{28}$ In their setting, manipulations under immediate acceptance reveal differences in preference intensities. In our case, theory predicts higher average payoffs under immediate acceptance because the lottery tends to increase truth-telling, yet $A$-types who were unsuccessful in the lottery at the most competitive school $A$ do not get to push out $B$-types who were admitted to $B$. The effect holds more generally, see Appendix B, Proposition 1.
} 
more similar schools than DA33 with respect to the average rank of the students. While there is no significant change in the difference in average ranks between the two most popular schools $A$ and $B$, the differences between them and the least preferred school are significantly lower under IA33. The difference in average ranks between the most preferred and the least preferred school, $\Delta_{A C}$, is 5.41 under DA33 and only 4.79 under IA33. Hence, in line with Hypothesis 6 we find:

Result 6. Comparing IA33 with DA33,

(i) the good-but-not-excellent are better off in terms of average payoffs and the probability of obtaining a seat at their preferred school under DA33, while the lower-ranked students are better off under IA33;

(ii) inequality as measured by the Gini index is lower under IA33 than under DA33; and

(iii) the difference between the average ranks at schools is smaller under IA33 than under DA33.

\section{Conclusions}

The paper is the first to study how to embed lotteries in school choice mechanisms with the aim to reduce disparities in the student composition between schools. We show that there are important differences between the effect of a lottery quota on the immediate acceptance mechanism (IA33) and the deferred acceptance mechanism (DA33).

Our experiment replicates previous evidence that truth-telling in the strategy-proof DA mechanism is significantly lower than predicted (see the survey by Hakimov and Kübler, 2019). More importantly, the experiment shows that a lottery quota increases truth-telling rates under the DA mechanism. This is in line with the theoretical result that a lottery quota reduces the set of equilibrium strategies under DA. Therefore, the increase in truth-telling under DA when combined with a lottery ensures that the application behavior provides valuable feedback for school authorities.

In equilibrium, DA, IA, and DA33 are stable while IA33 is not. In line with this, we observe that blocking pairs occur by far most frequently in IA33. While strategy-proofness and stability favor DA33 over IA33, the experiment also provides evidence that IA33 leads to more diverse schools and to more equitable outcomes across applicants of different priorities. Our theoretical results, both for the experimental markets at hand as well as for other quotas and more general profiles in a continuum model, reveal why this is the case. Good-but-not-excellent students fare worse under IA with a lottery since they are 
forced to choose between trying their luck in the lottery at a highly competitive school or relying on their relatively high grade priority at a less demanded school. In contrast, under DA with a lottery these students can still count on their priority to gain access at the less demanded school, should they be rejected by the competitive school. Conversely, lower-ranked students fare better under IA with a lottery.

With respect to strategy-proofness, stability, efficiency, equality of payoffs, and the desegregation of schools along the lines of academic achievement, none of the mechanisms dominates any other in all dimensions. The choice of a mechanism including a lottery quota by school authorities should depend on which properties are considered to be especially important.

\section{Appendix}

\section{A Proofs of Equilibrium Predictions}

\section{A.1 Equilibria under IA}

\begin{tabular}{l||c|c|c|c} 
students & $1-3$ & $4-6$ & $7-12(A)$ & $7-12(B)$ \\
\hline \hline match probability at $A$ & 1 & 0 & 0 & 0 \\
\hline match probability at $B$ & 0 & 1 & 0 & 0 \\
\hline match probability at $C$ & 0 & 0 & 1 & 1
\end{tabular}

Proof (Equilibria under IA). Since each school has at least three seats, the top three students 1-3 will be admitted wherever they apply first. Hence, for them not ranking $A$ first is strictly dominated. Given that $1-3$ will be matched to school $A$ in equilibrium, the three next-best students $4-6$ will be admitted either to $B$ or $C$ (or be unmatched), depending on where they apply first. Hence, for them it is a strict best response (to students $1-3)$ to rank $B$ first and be admitted. Since there are no other school seats available, for all remaining students $7-12$ it is a strict best response (to students $1-6$ ) to include school $C$ in their ranking and be admitted to $C$. 


\section{A.2 Equilibria under IA33}

\begin{tabular}{l||c|c|c|c} 
students & $1-2$ & $3-4$ & $5-12(A)$ & $7-12(B)$ \\
\hline \hline match probability at $A$ & 1 & 0 & 0.2 & 0 \\
\hline match probability at $B$ & 0 & 1 & 0 & 0.333 \\
\hline match probability at $C$ & 0 & 0 & 0.8 & 0.667
\end{tabular}

Proof (Equilibria under IA33). In equilibrium it is a strict best response for all students who are not certain of getting a seat at school $A$ or school $B$ to include school $C$ in their ranking. Furthermore, school $A$ allocates all its three seats in Step 1 of the IA33 algorithm, otherwise one of the nine $A$-type students could change his strategy, by reporting his type truthfully, and obtain a seat at school $A$. Similarly, school $B$ allocates all its three seats in Step 1 of the IA33 algorithm, otherwise one of the three $B$-type students could change his strategy, by reporting his most preferred school truthfully, and obtain a seat at school $B$.

This leaves school $C$ with at most six applicants in Step 1, so that each of them would be admitted if they included school $C$ in their ranking and received the second lowest payoff of 10 (the lowest payoff being 0 if they do not obtain a school seat). However, given the positive probability of receiving a more preferred school seat via the lottery, no one initially applies to school $C$ in equilibrium. All that remains to be shown is who initially applies to school $A$ and who initially applies to school $B$.

Given that students 1 and 2 will be admitted wherever they apply, not ranking $A$ first is strictly dominated. The two next best students 3 and 4 are guaranteed a seat at school $B$ if they apply there, which yields a payoff of 16 . If they were to apply to school $A$, they would enter a lottery for the one remaining seat, knowing that they can be admitted to $C$ if they lose. Hence, for students 3 or 4 to be willing to apply to school $A$ there would need to be at least a $50 \%$ chance of being admitted to $A$ (which yields a payoff of 22 , the expected payoff in the lottery with $50 \%$ is $1 / 2 \cdot 22+1 / 2 \cdot 10=16$ ) or at most four applicants at $A$ and thus at least eight applicants at $B$. However, then one of the worse-ranked $A$-type students applying to $B$ would deviate and apply to $A$ instead (receiving an expected payoff of $1 / 3 \cdot 22+2 / 3 \cdot 10=14$ instead of $1 / 6 \cdot 16+5 / 6 \cdot 10=11$ ). We conclude that in equilibrium, students 3 and 4 initially apply to $B$ and it is a strict best response for them to do so.

Next, we show that there are five applicants for the remaining lottery seat at school $A$ (so seven applicants at $A$, including students 1 and 2). Assume there were at most four applicants in the lottery at school $A$ and hence at least four in the lottery at school $B$. 
Then, there is at least one $A$-type student applying to $B$ where he receives an expected payoff of at most $1 / 4 \cdot 16+3 / 4 \cdot 10=11.5$. However, by applying to school $A$ instead, that $A$-type student would receive at least $1 / 5 \cdot 22+4 / 510=12.4$ and hence deviate. Assume, on the other hand, there were at least six applicants for the lottery at school $A$ and therefore at most two in the lottery at school $B$. There is then at least one $B$-type student applying to $A$ who could profitably deviate by applying to his more preferred and, at the same time, less competitive school.

Finally, knowing that there are five applicants in the lottery at school $A$ and three at school $B$, we also know that no $B$-type student will apply to $A$ because he would receive an expected payoff of $1 / 5 \cdot 16+4 / 5 \cdot 10=11.2$ while deviating and applying to $B$ would then yield $1 / 4 \cdot 22+3 / 4 \cdot 10=13$. Hence, all $B$-type students apply to school $B$ and include school $C$ in their ranking and all remaining $A$-type students (all $A$-type students ranked 5 and worse) apply to school $A$ and include school $C$ in their ranking. Finally, by the payoff comparisons in the preceding paragraph, these strategies are strict best responses.

\section{A.3 Equilibria under DA}

\begin{tabular}{l||c|c|c|c} 
students & $1-3$ & $4-6$ & $7-12(A)$ & $7-12(B)$ \\
\hline \hline match probability at $A$ & 1 & 0 & 0 & 0 \\
\hline match probability at $B$ & 0 & 1 & 0 & 0 \\
\hline match probability at $C$ & 0 & 0 & 1 & 1
\end{tabular}

Proof (Equilibria under $\boldsymbol{D A}$ ). Since each school has at least three seats, the top three students 1-3 will be admitted wherever they first apply. Hence, for them not ranking $A$ first is strictly dominated. Given that $1-3$ will be matched to school $A$ in equilibrium, the three next best students, 4-6, will be admitted either to $B$ or $C$ (or be unmatched), depending on which one they rank higher. Hence, for them it is a strict best response (to students 1-3) to rank $B$ first or second behind $A$ and be admitted to $B$. Since there are no other school seats available, for all remaining students $7-12$ it is a strict best response (to students 1-6) to include school $C$ in their ranking and be admitted to $C$. 


\section{A.4 Equilibria under DA33}

\begin{tabular}{l||c|c|c|c|c} 
students & $1-2$ & $3-4$ & 5 & $6-12(A)$ & $7-12(B)$ \\
\hline \hline match probability at $A$ & 1 & 0.133 & 0.133 & 0.133 & 0.022 \\
\hline match probability at $B$ & 0 & 0.867 & 0.324 & 0.086 & 0.2 \\
\hline match probability at $C$ & 0 & 0 & 0.543 & 0.781 & 0.778
\end{tabular}

Proof (Equilibria under DA33). Under the DA33 mechanism, it is a weakly dominant strategy for all students to report their true preferences (each student receives either a regular school seat based on his rank or a lottery seat based on his lottery rank independent of the step in the DA-algorithm; since matches are tentative until the very end of the algorithm, a student can safely report schools he might not match with without influencing later match chances).

By Fact 1, not ranking school $A$ first is strictly dominated for each student and for all but two students (students 1-2 are the exception) it even becomes a strict best response to report their true preferences.

Next, given that everybody reports their most preferred school truthfully, students 1 and 2 are admitted to school $A$. Next, we explain why all remaining $A$-type students $3-12(A)$ report their preferences truthfully in equilibrium: if a $3-12(A)$ student is ranked second by the lottery while some other $3-12(A)$ student is ranked first, he will be admitted to his second ranked school. Hence, it is strictly better to rank $B$ second and thereby truthfully reveal his preferences than to rank $C$ second (and not obtain $B$ with positive probability). Finally, since there is some probability of not being admitted to schools $A$ or $B$, students $3-12(A)$ rank school $C$ third.

Next, we explain why all $B$-type students $7-12(B)$ report their preferences truthfully in equilibrium: if a $7-12(B)$ student is ranked second by the lottery while some other 7-12(B) student is ranked first, he will be admitted to his second-ranked school - hence, it is strictly better to rank $A$ second and thereby truthfully reveal his preferences than to rank $C$ second (and not obtain $A$ with positive probability). Finally, since there is some probability of not being admitted to schools $A$ or $B$, students $7-12(B)$ rank school $C$ third.

When computing the resulting match probabilities, we need to take into account that the DA33 algorithm uses the same lottery ranking in all its steps. We can neglect the lottery ranking of students 1 and 2 who are admitted to $A$ based on their rank and focus only on the lottery ranking of the remaining students $3-12$. In the following, we consider five cases and calculate the associated conditional match probabilities. 
Case 1: With probability $2 / 10=1 / 5$ student 3 or student 4 has the best lottery rank. Conditional on this event, each of them is admitted to $A$ with probability $1 / 2$, while the other (3 or 4 ) and student 5 are admitted to $B$ based on rank. The remaining seven students are admitted to $B$ based on their lottery rank with equal probability $1 / 7$.

\begin{tabular}{l||c|c|c|c|c} 
students & $1-2$ & $3-4$ & 5 & $6-12(A)$ & $7-12(B)$ \\
\hline \hline match probability at $A$ & 1 & $1 / 2$ & 0 & 0 & 0 \\
\hline match probability at $B$ & 0 & $1 / 2$ & 1 & $1 / 7$ & $1 / 7$ \\
\hline match probability at $C$ & 0 & 0 & 0 & $6 / 7$ & $6 / 7$
\end{tabular}

Case 2: With probability $5 / 10=1 / 2$ an $A$-type student ranked $5-12$ (there are five such students) has the best lottery rank. Conditional on this event, each of them is admitted to $A$ with probability $1 / 5$, while students 3 and 4 are admitted to $B$ based on rank. The remaining seven students are admitted to $B$ based on their lottery rank with equal probability $1 / 7$.

\begin{tabular}{l||c|c|c|c|c} 
students & $1-2$ & $3-4$ & 5 & $6-12(A)$ & $7-12(B)$ \\
\hline \hline match probability at $A$ & 1 & 0 & $1 / 5$ & $1 / 5$ & 0 \\
\hline match probability at $B$ & 0 & 1 & $4 / 5 \cdot 1 / 7=4 / 35$ & $4 / 5 \cdot 1 / 7=4 / 35$ & $1 / 7$ \\
\hline match probability at $C$ & 0 & 0 & $24 / 35$ & $24 / 35$ & $6 / 7$
\end{tabular}

Case 3: With probability $3 / 10 \cdot 2 / 9=1 / 15$ a $B$-type student has the best lottery rank (there are three such students) and either student 3 or student 4 has the second best lottery rank. Conditional on this event, each of the $B$-type students is admitted to $B$ with probability $1 / 3$ and students 3 and 4 are each admitted to $A$ with probability $1 / 2$, while the other (3 or 4 ) and student 5 are admitted to $B$ based on rank. The remaining six students are admitted to $C$.

\begin{tabular}{l||c|c|c|c|c} 
students & $1-2$ & $3-4$ & 5 & $6-12(A)$ & $7-12(B)$ \\
\hline \hline match probability at $A$ & 1 & $1 / 2$ & 0 & 0 & 0 \\
\hline match probability at $B$ & 0 & $1 / 2$ & 1 & 0 & $1 / 3$ \\
\hline match probability at $C$ & 0 & 0 & 0 & 1 & $2 / 3$
\end{tabular}


Case 4: With probability $3 / 10 \cdot 5 / 9=1 / 6$ a $B$-type student (there are three such students) has the best lottery rank and an $A$-type student ranked 5-12 has the second best lottery rank (there are five such students). Conditional on this event, each of the $B$-type students is admitted to $B$ with probability $1 / 3$ and each of the $A$-type students ranked $5-12$ is admitted to $A$ with probability $1 / 5$, while students 3 and 4 are admitted to $B$ based on rank. The remaining six students are admitted to $C$.

\begin{tabular}{l||c|c|c|c|c} 
students & $1-2$ & $3-4$ & 5 & $6-12(A)$ & $7-12(B)$ \\
\hline \hline match probability at $A$ & 1 & 0 & $1 / 5$ & $1 / 5$ & 0 \\
\hline match probability at $B$ & 0 & 1 & 0 & 0 & $1 / 3$ \\
\hline match probability at $C$ & 0 & 0 & $4 / 5$ & $4 / 5$ & $2 / 3$
\end{tabular}

Case 5: With probability $3 / 10 \cdot 2 / 9=1 / 15$ a $B$-type student (there are three such students) has the best lottery rank and another $B$-type student has the second best lottery rank (there are two other such students). Conditional on this event, each of the $B$-type students is admitted to $B$ with probability $1 / 3$ and to $A$ with probability $1 / 3$, while students 3 and 4 are admitted to $B$ based on rank. The remaining six students are admitted to $C$.

\begin{tabular}{l||c|c|c|c|c} 
students & $1-2$ & $3-4$ & 5 & $6-12(A)$ & $7-12(B)$ \\
\hline \hline match probability at $A$ & 1 & 0 & 0 & 0 & $1 / 3$ \\
\hline match probability at $B$ & 0 & 1 & 0 & 0 & $1 / 3$ \\
\hline match probability at $C$ & 0 & 0 & 1 & 1 & $1 / 3$
\end{tabular}

Hence, adding up all probabilities, we obtain

\begin{tabular}{l||c|c}
\multicolumn{1}{c||}{ students } & $1-2$ & $3-4$ \\
\hline \hline match prob. at $A$ & $1 / 5+1 / 2+1 / 15+1 / 6+1 / 15=1$ & $1 / 10+1 / 30=2 / 15 \approx .133$ \\
\hline match prob. at $B$ & 0 & $1 / 10+1 / 2+1 / 30+1 / 6+1 / 15=13 / 15 \approx .867$ \\
\hline match prob. at $C$ & 0 & 0
\end{tabular}




\begin{tabular}{|c|c|c|}
\hline students & 5 & $6-12(A)$ \\
\hline match prob. at $\mathrm{A}$ & $1 / 10+1 / 30=2 / 15 \approx .133$ & $1 / 10+1 / 30=2 / 15 \approx .133$ \\
\hline match prob. at $B$ & $1 / 5+2 / 35+1 / 15=34 / 105 \approx .324$ & $1 / 35+2 / 35=3 / 35 \approx .086$ \\
\hline match prob. at $C$ & $12 / 35+2 / 15+1 / 15=57 / 105 \approx .543$ & $6 / 35+12 / 35+1 / 15+2 / 15+1 / 15=82 / 105 \approx .781$ \\
\hline
\end{tabular}

\begin{tabular}{l||c|c}
\multicolumn{1}{l||}{ students } & $7-12(B)$ & all students \\
\hline \hline match prob. at $A$ & $1 / 45 \approx .022$ & $2+4 / 15+2 / 15+8 / 15+1 / 15=3$ \\
\hline match prob. at $B$ & $1 / 35+1 / 14+1 / 45+1 / 18+1 / 45=1 / 5=.2$ & $26 / 15+34 / 105+12 / 35+3 / 5=3$ \\
\hline match prob. at $C$ & $6 / 35+3 / 7+2 / 45+1 / 9+1 / 45=7 / 9 \approx .778$ & $57 / 105+328 / 105+21 / 9=6$
\end{tabular}

\section{B Comparative Statics in a Continuum Model}

In this section, we show that a number of predictions for our experimental markets hold more generally. We present a continuum version of our school choice problem. This allows us to vary the lottery quota continuously and to investigate the comparative statics properties with respect to the distribution of payoffs and the student composition of schools. In the following we show that for any given lottery quota IA redistributes payoffs more strongly than DA (Proposition 1) and leads to more similar schools with respect to the average ranks of students (Proposition 2).

Consider a continuum of students with mass 1 , applying to three schools $A, B$, and $C$ with positive capacities $q_{A}, q_{B}$, and $q_{C}$, likewise of total mass 1 .

All schools have a common priority ranking over students that is based on academic achievement: each student $i$ 's priority $p(i)$ is drawn uniformly from the interval $[0,1]$ and student $i$ is considered to be of higher priority than student $j$ if and only if $p(i)<p(j)$.

All students consider school $C$ to be the least preferred school but may differ in whether they prefer school $A$ or school $B$ the most. Hence, there are two types of students: $a$-types have utilities $1=u_{a}(A)>u_{a}(B)>u_{a}(C)=0$ and $b$-types have utilities $1=u_{b}(B)>u_{b}(A)>u_{b}(C)=0$ (this generalizes the cardinal utilities chosen in our experimental school choice problems).

In our experimental school choice problems, preferences and priorities are correlated: all students of above median priority, $p(i) \leq \frac{1}{2}$, are $a$-types while the bottom half of students 
is split equally between $a$ - and $b$-types, making school $A$ both more popular and more competitive than school $B$. To generalize this preference profile, let us denote by $f_{A}(x)$ the fraction of students with priority $p(i)=x$ who are $a$-types, while $f_{B}(x)=1-f_{A}(x)$ is the corresponding fraction of $b$-types. We assume that school A is more competitive and more popular than school $B$ in that

(i) better students tend to prefer school $A$, i.e., $f_{A}(x)$ is weakly decreasing in $x$ and

(ii) even among the low priority students, school $A$ is still relatively more popular than school $B$, i.e.,

$$
\text { for all } x \in[0,1], \frac{f_{A}(x)}{q_{A}} \geq \frac{f_{B}(x)}{q_{B}} .
$$

Note that (ii), and $q_{C}>0$, imply that there are more $a$-types than seats at $A$ :

$$
\frac{\int_{0}^{1} f_{A}(x) \mathrm{d} x}{q_{A}} \geq \frac{\text { (ii) }}{\geq} \frac{\int_{0}^{1} f_{B}(x) \mathrm{d} x}{q_{B}} \stackrel{q_{C}>0}{>} \frac{1-\int_{0}^{1} f_{A}(x) \mathrm{d} x}{1-q_{A}} \Longrightarrow \int_{0}^{1} f_{A}(x) \mathrm{d} x>q_{A} .
$$

The continuum analogue to our experimental markets, satisfying (i) and (ii), would be $q_{A}=q_{B}=\frac{1}{4}, q_{C}=\frac{1}{2}$, and

$$
f_{A}(x)= \begin{cases}1, & \text { if } x \leq \frac{1}{2}, \text { and } \\ \frac{1}{2}, & \text { otherwise. }\end{cases}
$$

As mechanisms we consider variants of Immediate and Deferred Acceptance where a fraction $1-r$ of seats are allocated based on (academic achievement) priority while the remainder $r$ is allocated based on a random lottery ordering of students. For that, after students submit their strict preferences over schools, each student draws a lottery number $l(i)$, drawn uniformly from the interval $[0,1]$. For seats allocated by lottery, students with a lower lottery number are prioritized over those whose lottery number is higher. We refer to the deferred acceptance mechanism with lottery quota $r$ as DA- $r$ and to the immediate acceptance mechanism with lottery quota $r$ as IA- $r$.

\section{B.1 Equilibrium under DA-r}

The (truth-telling) equilibrium matching for DA-r is characterized by four cutoffs: $t_{A}^{D A}$ and $t_{B}^{D A}$ describe the priority cutoffs necessary to be admitted due to priority at school $A$ or $B$, while $l_{A}^{D A}$ and $l_{B}^{D A}$ describe the lottery cutoffs necessary to be admitted due to the lottery. The DA-r algorithm determines a matching and its associated cutoffs as follows.

Step 1. All $a$-types apply to school $A$ and all $b$-types to $B$. Since there are more $a$-types than seats at $A$, the school is oversubscribed. It (tentatively) admits all $a$-types with 
priority $p(i) \leq t_{A}^{D A}$ with cutoff $t_{A}^{D A}$ implicitly determined by

$$
\int_{0}^{t_{A}^{D A}} f_{A}(x) \mathrm{d} x=(1-r) q_{A}
$$

All remaining $a$-types enter the lottery at school $A$ and are (tentatively) admitted to $A$ with probability

$$
\frac{r \cdot q_{A}}{\int_{t_{A}^{D A}}^{1} f_{A}(x) \mathrm{d} x}=l_{A}^{D A} .
$$

School $B$ likewise (tentatively) admits applicants up to some cutoff $p(i) \leq \tilde{t}_{B}^{D A}$. If there are at most as many applicants as priority seats then $\tilde{t}_{B}^{D A}=1>t_{A}^{D A}$. If there are more applicants than priority seats, we also have $\tilde{t}_{B}^{D A} \geq t_{A}^{D A}$ : if not, then $\tilde{t}_{B}^{D A}<t_{A}^{D A}$ and $\int_{0}^{\tilde{t}_{B}^{D A}} f_{B}(x) \mathrm{d} x<\int_{0}^{t_{A}^{D A}} f_{B}(x) \mathrm{d} x \stackrel{\text { (ii) }}{\leq} \frac{\int_{0}^{t_{A}^{D A}} f_{A}(x) \mathrm{d} x}{q_{A}} \cdot q_{B}=\frac{\int_{0}^{t_{A}^{D A}} f_{A}(x) \mathrm{d} x}{(1-r) q_{A}} \cdot(1-r) q_{B}=(1-r) q_{B}$.

Hence, at cutoff $\tilde{t}_{B}^{D A}$ some of the $(1-r) q_{B}$ seats to be assigned based on priority at school $B$ are left unfilled; a contradiction.

Any remaining $b$-types enter the lottery and are (tentatively) admitted to $B$ with probability

$$
\tilde{l}_{B}^{D A}:=\min \left\{1, \frac{r \cdot q_{B}}{\int_{\tilde{t}_{B}^{D A}}^{1} f_{B}(x) \mathrm{d} x}\right\} \stackrel{\text { (ii) }}{\geq} \min \left\{1, \frac{r \cdot q_{A}}{\int_{\tilde{t}_{B}^{D A}}^{1} f_{A}(x) \mathrm{d} x}\right\} \geq \frac{r \cdot q_{A}}{\int_{t_{A}^{D A}}^{1} f_{A}(x) \mathrm{d} x}=l_{A}^{D A} .
$$

Step 2. Any $b$-types rejected at school $B$ apply to school $A$ and compete with the tentatively admitted $a$-types. However, as $t_{A}^{D A} \leq \tilde{t}_{B}^{D A}$ and $l_{A}^{D A} \leq \tilde{l}_{B}^{D A}$, they will be rejected once more, both for the priority and for the lottery seats, while the $a$-types admitted in Step 1 are admitted again.

Likewise, $a$-types rejected at $A$ (rejected both for priority and lottery seats) compete with the tentatively admitted $b$-types for priority seats at $B$. This creates a new priority cutoff $t_{B}^{D A} \geq t_{A}^{D A}$ given by

$$
\underbrace{\int_{0}^{t_{A}^{D A}} f_{B}(x) \mathrm{d} x}_{\leq(1-r) q_{B}, \text { by (ii) }}+\int_{t_{A}^{D A}}^{t_{B}^{D A}}\left(\left(1-l_{A}^{D A}\right) f_{A}(x)+f_{B}(x)\right) \mathrm{d} x=(1-r) q_{B} .
$$

Finally, consider $l_{B}^{D A}$, the cutoff determining the assignment of lottery seats at $B$. Toward a contradiction, assume $l_{B}^{D A}$ was smaller than $l_{A}^{D A}$. Then, $a$-types rejected at $A$ in Step 1 would stand no chance in the lottery and $l_{B}^{D A}$ would be determined by the applying $b$-types alone: those tentatively admitted in Step 1, i.e., with $p(i)>\tilde{t}_{B}^{D A}$ and $l(i) \leq \tilde{l}_{B}^{D A}$, as well 
as $b$-types who lost to $a$-types of higher priority in Step 2, i.e., with $p(i) \in\left(t_{B}^{D A}, \tilde{t}_{B}^{D A}\right]$ (and any $l(i))$. Since $b$-types tentatively admitted in Step 1 apply again, we have $l_{B}^{D A} \leq \tilde{l}_{B}^{D A}$. Hence, any applicant with $l(i)>\tilde{l}_{B}^{D A}$ who participates in the lottery stands no chance: his participation, or absence, leaves the cutoff $l_{B}^{D A}$ unaffected. Thus, we have

$$
l_{B}^{D A}=\frac{r \cdot q_{B}}{\int_{t_{B}^{D A}}^{1} f_{B}(x) \mathrm{d} x} \stackrel{(\mathrm{ii})}{\geq} \frac{r \cdot q_{A}}{\int_{t_{B}^{D A}}^{1} f_{A}(x) \mathrm{d} x} \geq \frac{r \cdot q_{A}}{\int_{t_{A}^{D A}}^{1} f_{A}(x) \mathrm{d} x}=l_{A}^{D A}
$$

which yields the desired contradiction to the assumption $l_{B}^{D A}<l_{A}^{D A}$.

Hence, we know that $t_{B}^{D A} \geq t_{A}^{D A}$ and $l_{B}^{D A} \geq l_{A}^{D A}$, so that all students rejected at school $B$ will also be rejected at school $A$ in Step 3 and will eventually be admitted to school $C$. Thus, the equilibrium matching is determined by cutoffs $t_{A}^{D A}, t_{B}^{D A}, l_{A}^{D A}$, and $l_{B}^{D A}$ where cutoffs $t_{B}^{D A}$ and $l_{B}^{D A}$ are jointly determined by

$$
q_{C}=\left(1-t_{B}^{D A}\right)\left(1-l_{B}^{D A}\right)
$$

Figure 4 illustrates the equilibrium matching graphically.
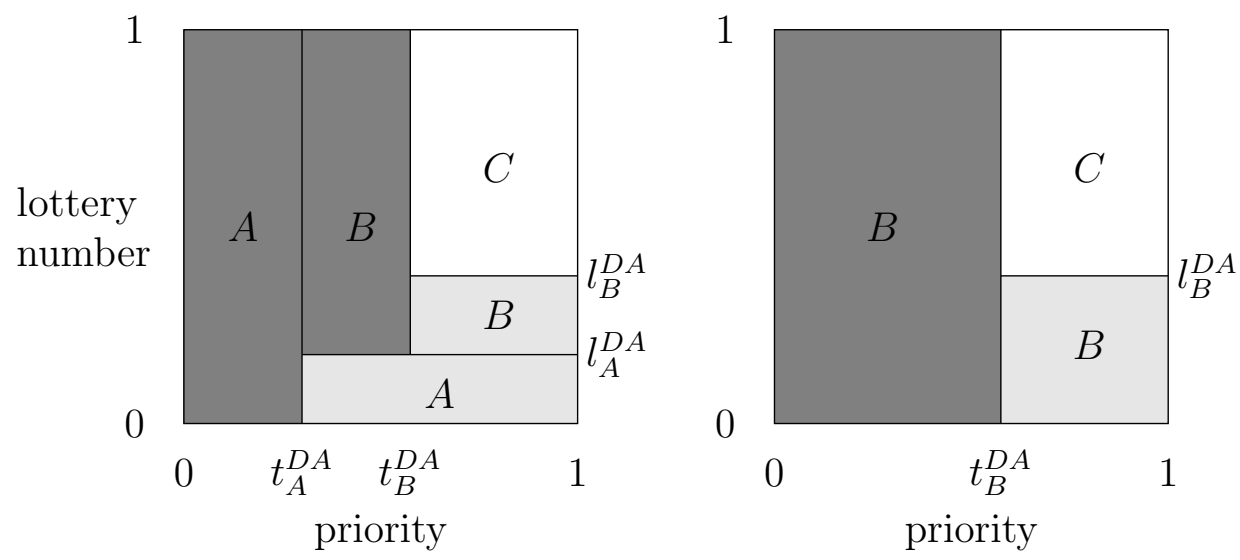

Figure 4: Equilibrium matching under DA-r.

Left: $a$-types.

Right: $b$-types.

Dark-gray: Seats assigned based on priority.

Light-gray: Seats assigned based on lottery number.

\section{B.2 Equilibrium under IA-r}

Given any submitted profile of preferences, the first step of the IA algorithm yields four cutoffs: $t_{A}^{I A}$ and $t_{B}^{I A}$ describe the priority cutoffs necessary to be admitted due to priority at school $A$ or $B$, while $l_{A}^{I A}$ and $l_{B}^{I A}$ describe the lottery cutoffs necessary to be admitted 
due to the lottery. Note that if there are fewer applicants than seats at a school in Step 1, then the cutoffs at that school can be set to 1 so that every applicant is admitted.

We will show that, in equilibrium, all (priority and lottery) quotas at schools $A$ and $B$ are filled completely in Step 1 of the IA-algorithm, provided that $a$-types have a sufficiently high valuation of $B$ :

\section{Condition 1.}

$$
u_{a}(B)>\frac{r\left(q_{A}+q_{B}\right)}{q_{C}+r\left(q_{A}+q_{B}\right)} \cdot{ }^{29}
$$

With both schools $A$ and $B$ filling their quotas completely in Step 1, the four cutoffs obtained in that step also determine the final matching so that, in equilibrium, each student's strategy is a best response to these cutoffs.

Identifying a best response is straightforward for the highest priority students, $a$-types with $p(i) \leq t_{A}^{I A}, b$-types with $p(i) \leq t_{B}^{I A}$ : they are certain of being admitted if and only if they apply to their most preferred school. We will show that $t_{A}^{I A} \leq t_{B}^{I A}$ and that the lottery at $B$ is less competitive, so that $b$-types with $p(i)>t_{B}^{I A}$ should rank school $B$ first. Low-priority $a$-types, those with $p(i)>t_{B}^{I A}$, will rank either $A$ or $B$ first, trading off a higher valuation of $A$ against a higher chance in the lottery at $B$. Finally, any good-but-not-excellent $a$-type student with $p(i) \in\left(t_{A}^{I A}, t_{B}^{I A}\right]$ could either rank school $A$ first to compete for a lottery seat or rank school $B$ first and be admitted with certainty; we will show that under Condition 1 , they prefer the latter which pins down $t_{B}^{I A}$.

Figure 5 illustrates the equilibrium matching graphically.

First, we show that, in equilibrium, schools $A$ and $B$ fill their quotas completely in Step 1 of the IA-algorithm. Since there are more $a$-types than seats at $A$, school $A$ will, in equilibrium, have at least as many applicants as seats in Step 1 (otherwise an $a$-type not ranking $A$ first could deviate and be admitted to $A$ for sure). Hence, $A$ fills its quota completely in Step 1. Likewise, $B$ fills its quota completely in Step 1 if there is a mass of at least $q_{B} b$-types.

Now, suppose that there are fewer $b$-types than seats at school $B$, i.e., $\int_{0}^{1} f_{B}(x) \mathrm{d} x<q_{B}$. We show that even then, given Condition $1, B$ will fill its quota in Step 1. Toward a contradiction, assume school $B$ had fewer applicants than seats in Step 1 . Then all $b$-types must have an expected utility of 1 , while all $a$ types must have an expected utility of at least $u_{a}(B)$. Moreover, $(1-r) q_{A} a$-types who are admitted to $A$ based on their priority have an expected utility of 1 . The aggregate welfare in equilibrium would thus be bounded

\footnotetext{
${ }^{29}$ For $q_{A}+q_{B}=\frac{1}{2}$, as in our experimental school choice problems, Condition 1 reduces to $u_{A}(B)>\frac{r}{1+r}$. Hence, for $u_{A}(B)=\frac{1}{2}$, which corresponds to our experimental payoffs, Condition 1 is satisfied for all lottery quotas $r \in[0,1)$.
} 

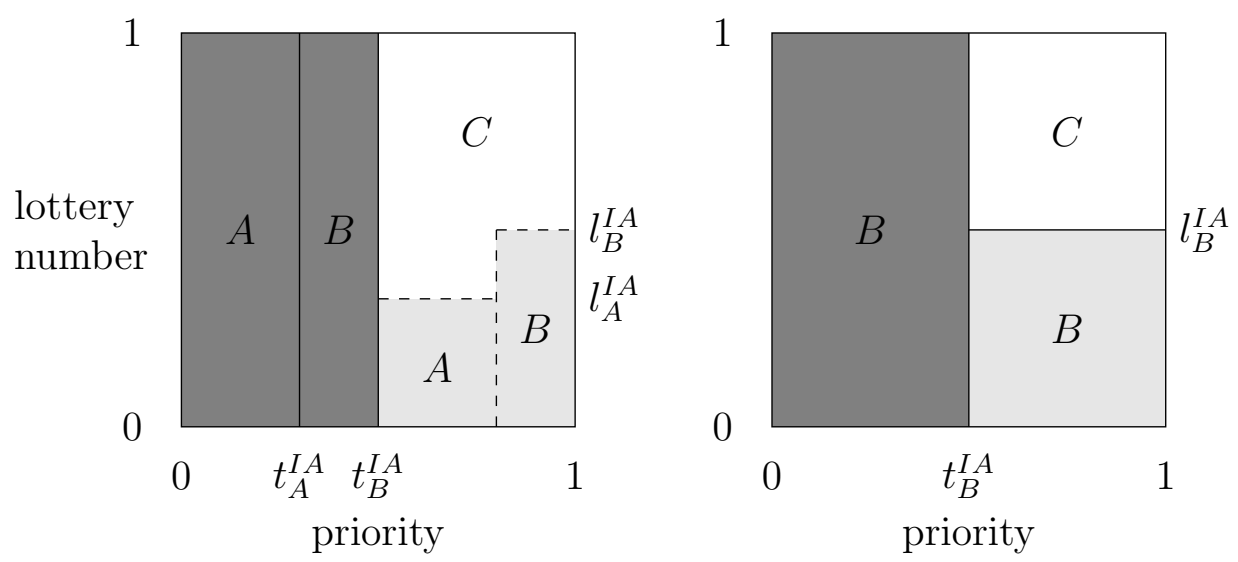

Figure 5: Equilibrium matching under IA-r.

Left: $a$-types

Right: $b$-types.

Dark-gray: Seats assigned based on priority.

Light-gray: Seats assigned based on lottery number.

Dashed: $a$-types with $p(i)>t_{B}^{I A}$ may be indifferent and enter lottery at either $A$ or $B$.

from below by

$$
\int_{0}^{1} f_{B}(x) \mathrm{d} x+(1-r) q_{A}+\left(\int_{0}^{1} f_{A}(x) \mathrm{d} x-(1-r) q_{A}\right) u_{a}(B) .
$$

In contrast, the largest feasible aggregate welfare arises when as many seats at each school as possible are filled with the respective type, i.e., all $q_{A}$ seats at $A$ are filled by $a$-types, $\int_{0}^{1} f_{B}(x) \mathrm{d} x$ seats at $B$ are filled by $b$-types, and the remaining seats at $B$ are filled by $a$-types:

$$
\int_{0}^{1} f_{B}(x) \mathrm{d} x+q_{A}+\left(q_{B}-\int_{0}^{1} f_{B}(x) \mathrm{d} x\right) u_{a}(B) .
$$

Taking the difference between the lower bound of equilibrium welfare and the maximal feasible welfare yields

$$
\begin{aligned}
-r \cdot q_{A}+\left(1-q_{A}-q_{B}+r \cdot q_{A}\right) u_{a}(B) & =-r \cdot q_{A}+\left(q_{C}+r \cdot q_{A}\right) u_{a}(B) \\
& \geq-r \cdot q_{A}+\left(q_{C}+r \cdot q_{A}\right) \frac{r\left(q_{A}+q_{B}\right)}{q_{C}+r\left(q_{A}+q_{B}\right)} \\
& >-r \cdot q_{A}+\left(q_{C}+r \cdot q_{A}\right) \frac{r \cdot q_{A}}{q_{C}+r \cdot q_{A}} \\
& =0,
\end{aligned}
$$

where the first inequality follows from Condition 1 and the second from $q_{B}>0$; a contradiction. We can now conclude that schools $A$ and $B$ fill their quotas completely in 
Step 1 of the IA-algorithm and that, hence, the cutoffs obtained in Step 1 determine the final assignment.

With $A$ filling its quota in Step 1, it is the unique best response for all $a$-types with $p(i) \leq t_{A}^{I A}$ to rank $A$ first (only then will they be admitted at $A$ ). Thus, $t_{A}^{I A}$ is given by

$$
\int_{0}^{t_{A}^{I A}} f_{A}(x) \mathrm{d} x=(1-r) q_{A}
$$

Likewise, $b$-types with $p(i) \leq t_{B}^{I A}$ apply to $B$ and are admitted with certainty. Since $A$ is assumed to be relatively more popular among (top priority) students (Assumption (ii)), we have $\int_{0}^{t_{A}^{I A}} f_{B}(x) \mathrm{d} x \leq(1-r) q_{B}$, and hence $t_{A}^{I_{A}} \leq t_{B}^{I_{A}}$.

Low-priority applicants with $p(i)>t_{B}^{I A}$, both $a$ - and $b$-types, enter the lottery at either $A$ or $B$. If the lottery at $B$ was more competitive, i.e., $l_{B}^{I A}<l_{A}^{I A}$, then all low-priority $a$-types would enter the lottery at $A$. But then the lottery at $A$ would be more competitive:

$$
l_{A}^{I A} \leq \frac{r \cdot q_{A}}{\int_{t_{B}^{I A}}^{1} f_{A}(x) \mathrm{d} x} \stackrel{\text { (ii) }}{\leq} \frac{r \cdot q_{B}}{\int_{t_{B}^{I A}}^{1} f_{B}(x) \mathrm{d} x} \leq l_{B}^{I A} ;
$$

a contradiction. Hence, $l_{B}^{I A} \geq l_{A}^{I A}$, so that all low-priority $b$-types enter the lottery at $B$.

Depending on $u_{a}(B)$, low-priority $a$-types may either all apply at $A$ (corner solution) or mix between ranking $A$ and $B$ first, with $l_{A}^{I A}$ and $l_{B}^{I A}$ adjusting accordingly so that they are indifferent. Good-but-not-excellent $a$-types with $p(i) \in\left(t_{A}^{I A}, t_{B}^{I A}\right]$ may enter the lottery at $A$, or apply at $B$ and are certain of being admitted. In either case we know that

$$
\underbrace{1-(1-r)\left(q_{A}+q_{B}\right)}_{\text {all students competing in a lottery }}=\underbrace{\frac{r \cdot q_{A}}{l_{A}^{I A}}}_{\text {at lottery for } A}+\underbrace{\frac{r \cdot q_{B}}{l_{B}^{I A}}}_{\text {at lottery for } B} .
$$

Together with $l_{A}^{I A} \leq l_{B}^{I A}$ this yields

$$
l_{A}^{I A} \leq \frac{r\left(q_{A}+q_{B}\right)}{1-(1-r)\left(q_{A}+q_{B}\right)} \leq l_{B}^{I A}
$$

Hence, Condition 1 ensures that

$$
u_{a}(B)>\frac{r\left(q_{A}+q_{B}\right)}{q_{C}+r\left(q_{A}+q_{B}\right)}=\frac{r\left(q_{A}+q_{B}\right)}{1-(1-r)\left(q_{A}+q_{B}\right)} \geq l_{A}^{I A}
$$

so that $a$-types indeed prefer to be admitted to $B$ with certainty over entering the lottery at $A$. Thus good-but-not-excellent $a$-types with $p(i) \in\left(t_{A}^{I A}, t_{B}^{I A}\right]$ apply to $B$ so that $t_{B}^{I A}$ is 
given by

$$
\int_{0}^{t_{B}^{I A}} f_{A}(x)+f_{B}(x) \mathrm{d} x=t_{B}^{I A}=(1-r)\left(q_{A}+q_{B}\right) .
$$

\section{B.3 Comparative Statics with Respect to the Lottery Quota}

Under both mechanisms, an increase in the lottery quota $r$ redistributes from high to low priority applicants in that it lowers the priority cutoffs (fewer admissions via high priority) and increases the lottery cutoffs (better chances in the lottery for low priority applicants).

\section{Comparative Statics DA-r}

For DA- $r$, the priority cutoff $t_{A}^{D A}$ is decreasing in $r$ as follows from its implicit specification in Section B.1:

$$
\int_{0}^{t_{A}^{D A}} f_{A}(x) \mathrm{d} x=(1-r) q_{A} .
$$

The lottery cutoff $l_{A}^{D A}$ is increasing in $r$ since

$$
l_{A}^{D A}=\frac{r \cdot q_{A}}{\int_{t_{A}^{D A}}^{1} f_{A}(x) \mathrm{d} x}=\frac{r \cdot q_{A}}{\int_{0}^{1} f_{A}(x) \mathrm{d} x-\int_{0}^{t_{A}^{D A}} f_{A}(x) \mathrm{d} x}=\frac{r \cdot q_{A}}{\underbrace{\int_{0}^{1} f_{A}(x) \mathrm{d} x-q_{A}}_{\text {constant, }>0}+r \cdot q_{A}} .
$$

For the cutoffs determining admission to $B$, we know that they satisfy

$$
q_{C}=\left(1-t_{B}^{D A}\right)\left(1-l_{B}^{D A}\right)
$$

Hence, if $t_{B}^{D A}$ were to increase as $r$ increases, then $l_{B}^{D A}$ would have to decrease and vice versa. Moreover with $r \cdot q_{B}$ seats at $B$ assigned via lottery, the cutoff $l_{B}^{D A}$ satisfies the following condition (see Figure 4: adding up the two light-gray lottery rectangles labelled as school $B)$ :

$$
r \cdot q_{B}=\int_{t_{B}^{D A}}^{1}\left(l_{B}^{D A}-l_{A}^{D A}\right) f_{A}(x) \mathrm{d} x+\int_{t_{B}^{D A}}^{1} l_{B}^{D A} \cdot f_{B}(x) \mathrm{d} x .
$$

Recall that $l_{A}^{D A}$ is increasing in $r$, so if $t_{B}^{D A}$ was increasing in $r$ and hence $l_{B}^{D A}$ decreasing, then the right-hand side would be decreasing in $r$; a contradiction. We conclude the $t_{B}^{D A}$ is decreasing in $r$ and $l_{B}^{D A}$ is increasing in $r$. 


\section{Comparative Statics IA-r}

For IA- $r$, the priority cutoffs $t_{A}^{I A}$ and $t_{B}^{I A}$ are decreasing in $r$ as follows from their implicit specifications in Section B.2:

$$
\int_{0}^{t_{A}^{I A}} f_{A}(x) \mathrm{d} x=(1-r) q_{A} \text { and } t_{B}^{I A}=\int_{0}^{t_{B}^{I A}} f_{A}(x)+f_{B}(x) \mathrm{d} x=(1-r)\left(q_{A}+q_{B}\right) .
$$

Depending on $u_{a}(B)$, low-priority $a$-types, $p(i)>t_{B}^{I A}$, may either split between entering the lottery at $A$ and $B$ (interior solution) or they may all enter the lottery at $A$ (corner solution). Let us first consider an interior solution. It requires low-priority $a$-types to be indifferent between the two options, which fixes the ratio between the two cutoffs: $l_{A}^{I A}=u_{a}(B) \cdot l_{B}^{I A}$. Thus, either both cutoffs are increasing in $r$ (for a marginal increase of $r$ ), or both are decreasing. Moreover, with the ratio of $l_{A}^{I A}$ and $l_{B}^{I A}$ fixed by $u_{a}(B)$, so is the ratio of low-priority applicants, $p(i)>t_{B}^{I A}$, that enter the lottery at $A$ and $B$. Then if both lottery cutoffs (as well as both priority cutoffs) were decreasing in $r$, the mass of students admitted to $C$ would be increasing in $r$ (see Figure 5). But since that mass is constant, we conclude that both lottery cutoffs are increasing in $r$. Analogously, one can show that the lottery cutoffs are increasing in $r$ in the case of a corner solution where all $a$-types with $t>t_{B}^{I A}$ enter the lottery at $A$.

\section{B.4 Comparing DA- $r$ and IA- $r$}

First, our analysis in Sections B.1 and B.2 reveals that the priority cutoff for school $A$ is the same under both mechanisms,

$$
t_{A}=t_{A}^{D A}=t_{A}^{I A}
$$

For the priority cutoffs $t_{B}^{D A}$ and $t_{B}^{I A}$, consider the dark-gray rectangles in Figures 4 and 5 labelled school $B$. Formally, the mass of students covered by these rectangles equals $(1-r) q_{B}$ and corresponds to the following integrals on the right hand side:

$$
\begin{aligned}
\underbrace{(1-r) q_{B}}_{\text {priority seats at } B} & \left.=\int_{0}^{t_{A}} f_{B}(x) \mathrm{d} x+\int_{t_{A}}^{t_{B}^{I_{A}}} f_{A}(x)+f_{B}(x) \mathrm{d} x \text { (at IA- } r \text {, see Figure } 5\right) \\
& =\int_{0}^{t_{A}} f_{B}(x) \mathrm{d} x+\int_{t_{A}}^{t_{B}^{D A}} \underbrace{\left(1-l_{A}^{D A}\right) f_{A}(x)}_{<f_{A}(x)}+f_{B}(x) \mathrm{d} x \text { (at DA-r, see Figure } 4) .
\end{aligned}
$$


Hence, with a smaller integrand in the case of DA- $r$, the bounds of integration have to expand: $t_{B}^{D A}>t_{B}^{I A}$.

For the lottery cutoffs for school $B$, we have

$$
\begin{aligned}
l_{B}^{I A} \geq \frac{r\left(q_{A}+q_{B}\right)}{1-(1-r)\left(q_{A}+q_{B}\right)} & =\frac{1-(1-r)\left(q_{A}+q_{B}\right)-\left(1-q_{A}-q_{B}\right)}{1-(1-r)\left(q_{A}+q_{B}\right)} \\
& =1-\frac{q_{C}}{1-t_{B}^{I A}} \\
& >1-\frac{q_{C}}{1-t_{B}^{D A}} \\
& =l_{B}^{D A} .
\end{aligned}
$$

Finally, the lottery cutoff for school $A$ is higher under IA- $r$ than under DA- $r$ since under IA-r only a subset of $a$-types enters the lottery for $A$ :

$$
l_{B}^{I A}>l_{B}^{D A}
$$

We now show that good-but-not-excellent students are worse off under IA-r than under DA- $r$, while low-priority students fare better under IA- $r$ than under DA- $r$.

Proposition 1. For any lottery size $r \in(0,1)$ and given Condition 1, IA-r yields higher aggregate welfare than DA-r. Moreover, students with priority $p(i) \leq t_{B}^{D A}$ either receive the same match or are weakly worse off under IA-r compared to DA-r. Lower priority students with priority $p(i)>t_{B}^{D A}$ are, on average, better off in IA-r.

Proof. Aggregate welfare is maximized if all seats at school $A$ are occupied by $a$-types and all seats at school $B$ by $b$-types. As $A$ is exclusively filled with $a$-types under both mechanisms, aggregate welfare only depends on the number of seats at $B$ filled by $b$-types. Put differently, aggregate welfare is higher when fewer $b$-types enter school $C$, namely for IA- $r$ :

$$
\begin{aligned}
\underbrace{\left(1-l_{B}^{D A}\right) \int_{t_{B}^{D A}}^{1} f_{B}(x) \mathrm{d} x}_{b \text {-types at } C \text { for DA- } r} & =\frac{q_{C}}{1-t_{B}^{D A}} \int_{t_{B}^{D A}}^{1} f_{B}(x) \mathrm{d} x \\
& >\frac{q_{C}}{1-t_{B}^{I A}} \int_{t_{B}^{I A}}^{1} f_{B}(x) \mathrm{d} x \\
& \geq \underbrace{\left(1-l_{B}^{I A}\right) \int_{t_{B}^{I A}}^{1} f_{B}(x) \mathrm{d} x}_{b \text {-types at } C \text { for IA- } r}
\end{aligned}
$$

where the first inequality follows from the fact that $t_{B}^{D A}>t_{B}^{I A}$ and $f_{B}(x)$ is weakly 
increasing in $x$ while the second follows from our bound on $l_{B}^{I A}$, see $\left(1: l_{B}^{I A} \geq 1-\frac{q_{C}}{1-t_{B}^{I A}}\right)$.

Next, consider students of different priorities. High priority students ( $a$-types with $p(i) \leq t_{A}^{I A}=t_{A}^{D A}$ and $b$-types with $\left.p(i) \leq t_{B}^{I A}\right)$ are admitted to their most preferred school under both mechanisms. Next, $a$-types with priority $p(i) \in\left(t_{A}^{I A}, t_{B}^{I A}\right]$ are worse off under IA- $r$ : they are admitted to $B$ when they had a positive probability of being admitted to $A$ under DA-r (and were otherwise admitted to $B$ ). For students with priority $p(i) \in\left(t_{B}^{I A}, t_{B}^{D A}\right]$, both types are worse off: $b$-types were certain of being admitted to $B$ under DA- $r$ while they face a lottery between $B$ and $C$ under IA- $r$; $a$-types enter a lottery under IA- $r$ when they would prefer $B$ for sure and would do even better than that under DA- $r$ where they face a lottery between $A$ and $B$.

Finally, given that aggregate welfare is weakly higher under IA- $r$ than under DA- $r$ but all students with high enough priority $p(i) \leq t_{B}^{D A}$ are weakly worse off (some strictly), we know that lower priority students with priority $p(i)>t_{B}^{D A}$ are, on average, better off.

While low priority students are, on average, better-off under IA- $r$ than under DA- $r$, the welfare gains may be unevenly distributed between $a$ - and $b$-types; while the latter are always strictly better off (they have a higher probability of being admitted to $B$ and a lower probability of being admitted to $C$ ), the welfare effect on $a$-types is ambiguous and depends on $u_{a}(B)$ as well as on the distribution of both types. However, if $b$-types are not too numerous, low-priority $a$-types are also strictly better off. In particular this is the case for aligned preferences:

Corollary 1. Given Condition 1, if all students are a-types, then for any lottery quota $r \in(0,1)$,

- students with priority $p(i) \leq t_{A}^{I A}$ are always admitted to $A$ and hence are indifferent between DA-r and IA-r,

- students with priority $p(i) \in\left(t_{A}^{I A}, t_{B}^{D A}\right]$ fare worse under $I A-r$,

- students with priority $p(i)>t_{B}^{D A}$ fare better under IA-r.

Proof. In the absence of $b$-types, since all students have identical utility functions, aggregate welfare is independent of the matching. High priority students are admitted to $A$ under both mechanisms, while students of priority $p(i) \in\left(t_{A}^{I A}, t_{B}^{I A}\right]$ are admitted to $B$ under IA- $r$ (instead of facing a lottery between $A$ and $B$ under DA-r); hence they fare worse under IA- $r$. For the next lowest priority students with priorities $p(i) \in\left(t_{B}^{I A}, t_{B}^{D A}\right]$, it gets even worse under IA- $r$ as they now have to enter a lottery when they would prefer to be admitted to $B$ for sure and would do even better under DA- $r$ where they enter a lottery between $A$ and $B$. Hence, all students of lower priority (who all receive the same 
expected utility as they face the same lottery cutoffs $l_{A}^{I A}$ and $l_{B}^{I A}$ and are in equilibrium indifferent between applying at $A$ or $B$ ) are the ones who fare better under IA-r than under DA- $r$.

Besides affecting individual welfare, both mechanisms also generate different student distributions at the schools. In particular, under DA- $r$, school $A$ will, on average, admit students with the highest priorities, $C$, on average, those with the lowest priorities. Next, we show that IA-r reduces this gap: it lowers the average priority at $A$ and increases the average priority at $C$. In particular, let us assume that if under IA- $r$ (low-priority) $a$-types are indifferent between entering the lottery at $A$ or $B$ then they all mix between the two options with equal probabilities (i.e., we consider an equilibrium where we restrict all $a$-types with priorities below $t_{B}^{I A}$ to the same strategy).

Proposition 2. For any lottery size $r \in(0,1)$ and given Condition 1,

- the distribution of student priorities at school A under DA-r first-order stochastically dominates the corresponding distribution under IA-r (i.e., A admits students of lower priorities under IA-r),

- the distribution of student priorities at school $C$ under IA-r first-order stochastically dominates the corresponding distribution under DA-r (i.e., C admits students of higher priority under $I A-r)$.

Proof. Let us first consider school $A$ which is exclusively filled with $a$-types under both mechanisms. The set of students admitted based on priority is also identical in both cases: all $a$-types with priority $p(i) \leq t_{A}=t_{A}^{I A}=t_{A}^{D A}$. However, the $r \cdot q_{A}$ students admitted via lottery differ: under DA- $r$, these are $a$-types from the interval $\left(t_{A}, 1\right]$ with each such student admitted with probability $l_{A}^{D A}$, while under IA- $r$ no good-but-not-excellent $a$-type from the priority interval $\left(t_{A}, t_{B}^{I A}\right]$ is admitted. Instead, there is a corresponding increase in the probability with which a low-priority $a$-type from the priority interval $\left(t_{B}^{I A}, 1\right] \subset\left(t_{A}^{D A}, 1\right]$ is admitted.

Next, consider school $C$. Under DA-r all students with priorities $p(i) \in\left(t_{B}^{D A}, 1\right]$ are admitted to $C$ with equal probability $1-l_{B}^{D A}$. Under IA- $r$, students with priorities $p(i) \in\left(t_{B}^{I A}, 1\right] \supset\left(t_{B}^{D A}, 1\right]$ are admitted to $C$ : $b$-types with probability $1-l_{B}^{I A}, a$-types with a probability between $1-l_{A}^{I A}$ and $1-l_{B}^{I A}$ (depending on the probability with which they mix between applying to $A$ and $B$ ). Thus, if the fraction of $a$-types, $f_{A}(x)$, was constant over the interval $\left(t_{B}^{I A}, 1\right]$, priorities of students admitted to $C$ would be uniformly distributed over the interval $\left(t_{B}^{I A}, 1\right]$ and hence, of higher priority (lower $p(i)$ ) than under DA- $r$ where priorities of admitted students are uniformly distributed over the subset $\left(t_{B}^{D A}, 1\right] \subset\left(t_{B}^{I A}, 1\right]$. Moreover, if $f_{A}(x)$ is decreasing (so that among low priority students, 
those with relatively higher priority are more likely to prefer $A$ ), and as under IA- $r$ the probability of $a$-types to be assigned to $C$ is higher than that of $b$-types, this only further increases the distribution of priorities at $C$ under IA- $r$.

\section{Additional Tables}

Table 10: Sources of Blocking Pairs

\begin{tabular}{c|c|cccc} 
& Blocked by & IA & DA & IA33 & DA33 \\
\hline \multirow{3}{*}{ school A } & rank criterion & 43 & 15 & 8 & 12 \\
& lottery criterion & - & - & 213 & 76 \\
& both criteria & - & - & 2 & 2 \\
\hline \multirow{3}{*}{ school B } & rank criterion & 73 & 14 & 118 & 5 \\
& lottery criterion & - & - & 105 & 44 \\
& both criteria & - & - & 26 & 1 \\
\hline \multirow{3}{*}{ school C } & rank criterion & 4 & 1 & 10 & 4 \\
& lottery criterion & - & - & 10 & 4 \\
& both criteria & - & - & 10 & 4
\end{tabular}

Notes: The table displays the number of blocking pairs and whether a school and a student block the matching for a lottery seat or for a seat allocated according to the students' grade rank.

Table 11: Number of Blocking Pairs

\begin{tabular}{l||c|c|c|c}
$i$ & IA & DA & IA33 & DA33 \\
\hline \hline 1 & 7 & 2 & 0 & 4 \\
2 & 3 & 3 & 7 & 6 \\
3 & 6 & 8 & 78 & 15 \\
4 & 35 & 8 & 53 & 16 \\
5 & 23 & 5 & 51 & 14 \\
6 & 19 & 2 & 41 & 10 \\
8 & 7 & 1 & 34 & 16 \\
9 & 5 & 0 & 26 & 15 \\
10 & 3 & 0 & 44 & 9 \\
11 & 0 & 0 & 32 & 13 \\
12 & 1 & 0 & 29 & 10 \\
\hline total & 120 & 30 & 426 & 138 \\
\hline
\end{tabular}

Notes: The table displays the total number of blocking pairs for each student type across the four mechanisms. 


\section{Documentation of the Experiment}

\section{D.1 Preference Profiles}

Table 12 displays the 20 preference profiles used for all groups in the order indicated in the table.

Table 12: Preference Profiles of the Experimental School Choice Problems

\begin{tabular}{l||c|c|c|c|c|c|c|c|c|c|c|c|c|c|c|c|c|c|c|c}
$i$ & $P 1$ & $P 2$ & $P 3$ & $P 4$ & $P 5$ & $P 6$ & $P 7$ & $P 8$ & $P 9$ & $P 10$ & $P 11$ & $P 12$ & $P 13$ & $P 14$ & $P 15$ & $P 16$ & $P 17$ & $P 18$ & $P 19$ & $P 20$ \\
\hline \hline 1 & $A$ & $A$ & $A$ & $A$ & $A$ & $A$ & $A$ & $A$ & $A$ & $A$ & $A$ & $A$ & $A$ & $A$ & $A$ & $A$ & $A$ & $A$ & $A$ & $A$ \\
2 & $A$ & $A$ & $A$ & $A$ & $A$ & $A$ & $A$ & $A$ & $A$ & $A$ & $A$ & $A$ & $A$ & $A$ & $A$ & $A$ & $A$ & $A$ & $A$ & $A$ \\
3 & $A$ & $A$ & $A$ & $A$ & $A$ & $A$ & $A$ & $A$ & $A$ & $A$ & $A$ & $A$ & $A$ & $A$ & $A$ & $A$ & $A$ & $A$ & $A$ & $A$ \\
4 & $A$ & $A$ & $A$ & $A$ & $A$ & $A$ & $A$ & $A$ & $A$ & $A$ & $A$ & $A$ & $A$ & $A$ & $A$ & $A$ & $A$ & $A$ & $A$ & $A$ \\
5 & $A$ & $A$ & $A$ & $A$ & $A$ & $A$ & $A$ & $A$ & $A$ & $A$ & $A$ & $A$ & $A$ & $A$ & $A$ & $A$ & $A$ & $A$ & $A$ & $A$ \\
6 & $A$ & $A$ & $A$ & $A$ & $A$ & $A$ & $A$ & $A$ & $A$ & $A$ & $A$ & $A$ & $A$ & $A$ & $A$ & $A$ & $A$ & $A$ & $A$ & $A$ \\
7 & $A$ & $A$ & $A$ & $A$ & $A$ & $A$ & $A$ & $A$ & $A$ & $A$ & $B$ & $B$ & $B$ & $B$ & $B$ & $B$ & $B$ & $B$ & $B$ & $B$ \\
8 & $A$ & $A$ & $A$ & $A$ & $B$ & $B$ & $B$ & $B$ & $B$ & $B$ & $A$ & $A$ & $A$ & $A$ & $A$ & $A$ & $B$ & $B$ & $B$ & $B$ \\
9 & $A$ & $B$ & $B$ & $B$ & $A$ & $A$ & $A$ & $B$ & $B$ & $B$ & $A$ & $A$ & $A$ & $B$ & $B$ & $B$ & $A$ & $A$ & $A$ & $B$ \\
10 & $B$ & $A$ & $B$ & $B$ & $A$ & $B$ & $B$ & $A$ & $A$ & $B$ & $A$ & $B$ & $B$ & $A$ & $A$ & $B$ & $A$ & $A$ & $B$ & $A$ \\
11 & $B$ & $B$ & $A$ & $B$ & $B$ & $A$ & $B$ & $A$ & $B$ & $A$ & $B$ & $A$ & $B$ & $A$ & $B$ & $A$ & $A$ & $B$ & $A$ & $A$ \\
12 & $B$ & $B$ & $B$ & $A$ & $B$ & $B$ & $A$ & $B$ & $A$ & $A$ & $B$ & $B$ & $A$ & $B$ & $A$ & $A$ & $B$ & $A$ & $A$ & $A$ \\
\hline
\end{tabular}

Notes: Preference types (most preferred schools) of students 1-12 in profiles $P 1-P 20$.

\section{D.2 Instructions and Quizzes}

We only provide the instructions and the quiz for treatments IA33 and DA33. The instructions and quiz for IA and DA are available from the authors.

\section{Instructions for Treatment IA33 (translated from German)}

The experiment you are about to participate in is part of a project funded by the Deutsche Forschungsgemeinschaft (DFG) to analyze decision-making processes. In this experiment, you can earn a considerable amount of money, depending on your decisions and the decisions of the other participants. It is therefore essential that you read the instructions carefully.

Please do not speak to the other participants. If you have a question, simply raise your hand. We will then come to you and answer your question discreetly. Please do not ask your question(s) in public. 


\section{Decision Situation}

In this experiment, we simulate a procedure that matches places at schools to students. In this procedure, students apply to a central clearinghouse that allocates the students to the schools. You and the other participants take on the role of the students.

\section{Procedure during the Experiment}

At the beginning of the experiment, you and the other participants will be divided into groups of 12 students each.

After you and the other participants have read the instructions, everyone will be asked to answer five short control questions. With these questions, we will test your understanding of the procedure and of the decisions you have to make. One of the experimenters will come to you and will go through all the answers with you. This will give you the opportunity to ask any remaining questions.

You and the other 11 students are applying to the central clearinghouse for a school seat. Schools $A$ and $B$ each have three seats to offer, school $C$ is offering 6 seats. In total, there are 12 school seats available to you and the other 11 participants. A computer program, which is explained in further detail below, determines who gains a seat at which school depending on the applications sent by you and the other participants. You will then be told whether your application was successful and which school seats you and the other participants of your group received.

The experiment consists of 24 rounds. In every round, the application procedure starts anew, and each round is independent from the others. This means that whether you get a seat or not and at which school you are admitted only depends on your application in this round. Your chances in the current round are therefore independent of the decisions that you and the other participants took in previous rounds.

\section{Payoffs}

At the end of the experiment, one round will be picked at random to determine your payoff. This payoff depends on the school you were admitted to in that particular round. Additionally, you will receive $5 €$ for your participation in the experiment.

At the beginning of each round, a rank (indicating your ranking in terms of school grades in relation to the other students) is assigned to you and a payoff table is shown. The table shows which amount every participant gets for a seat at a certain school. Every participant knows the payoff table for the round. In every round, the amount of money you get for being admitted to a certain school is determined anew, and you are assigned a new rank. The rank is shown in the upper-left corner of your screen. 
To help you familiarize yourself with the structure of the payoff table, we will now show you an example.

Table 13: Payoff Table

\begin{tabular}{l||c|c|c} 
& School $A$ & School $B$ & School $C$ \\
\hline \hline Payoff of the student in rank 1 & 22 Euro & 16 Euro & 10 Euro \\
Payoff of the student in rank 2 & 22 Euro & 16 Euro & 10 Euro \\
Payoff of the student in rank 3 & 22 Euro & 16 Euro & 10 Euro \\
Payoff of the student in rank 4 & 22 Euro & 16 Euro & 10 Euro \\
Payoff of the student in rank 5 & 22 Euro & 16 Euro & 10 Euro \\
Payoff of the student in rank 6 & 22 Euro & 16 Euro & 10 Euro \\
Payoff of the student in rank 7 & 22 Euro & 16 Euro & 10 Euro \\
Payoff of the student in rank 8 & 22 Euro & 16 Euro & 10 Euro \\
Payoff of the student in rank 9 & 22 Euro & 16 Euro & 10 Euro \\
Payoff of the student in rank 10 & 16 Euro & 22 Euro & 10 Euro \\
Payoff of the student in rank 11 & 16 Euro & 22 Euro & 10 Euro \\
Payoff of the student in rank 12 & 16 Euro & 22 Euro & 10 Euro \\
\hline
\end{tabular}

Notes: Example of a payoff table for all participants in your group. [The German instructions show the screenshot of this page.]

This table can be read as follows: Assume that you are the student ranked 6th. This means that you get $10 €$ if you are admitted to school $C$ and this round is chosen for the payoff. Accordingly, you get $16 €$ if you are admitted to school $B$ or $22 €$ if you are admitted to school $A$. If you are not admitted to any school, you will receive $0 €$. In either case, you will receive an additional $5 €$ for your participation in the experiment.

Important: The payoff table shown above only serves as an example. It is not connected in any way to your situation in the actual experiment.

\section{Possible Choices}

In each round, your seat at one of the schools will be determined according to the following procedure: You apply to the schools by creating a rank-order list of the schools according to your preference. On that list, you can state which school is your first, your second, and your third preference. You will see the following boxes to list your choices in each round: 


\begin{tabular}{lr|ll|ll}
\multicolumn{2}{c|}{ First Preference } & \multicolumn{2}{|c|}{ Second Preference } & \multicolumn{2}{|c}{ Third Preference } \\
\hline \hline School $A$ & $\square$ & School $B$ & $\square$ & School $C$ & $\square$ \\
School $A$ & $\square$ & School $B$ & $\square$ & School $C$ & $\square$ \\
School $A$ & $\square$ & School $B$ & $\square$ & School $C$ & $\square$ \\
\hline
\end{tabular}

Notes: Boxes to indicate your preference. [The German instructions show the screenshot of this page.]

You are free to choose which school you will list as your first, second, and third preference. You are also free to decide whether to list one, two, all three, or none of the schools. For each choice, you can only indicate one school, and each school can only be listed once. You can only be admitted to the schools which you have explicitly indicated in your preference list.

Please make a choice for each preference and then press the button "My choice."

After all participants have entered their preference lists, the computer program will determine the school seats that you and the other participants in your group will receive. You will then see at which school you are admitted or whether you were not admitted at all. You will also see which schools the other participants have been admitted to. The allocation procedure that determines your rank is explained in detail below. Please read the rules of the allocation procedure carefully!

\section{The Allocation Procedure}

In each round, you and the other students in your group will be randomly assigned a rank. The rank varies between 1 and 12, with each of the students occupying exactly one rank. Rank 1 means that the student has the best grades, rank 12 means that the student has the worst grades. Schools prefer students with better grades over students with worse grades.

The allocation of the participants to the school seats works as follows:

\section{Round 1:}

- Each student applies to the school he listed as his first preference.

- If fewer or exactly as many students as there are seats at a school have listed that school as their first preference, all of them get a seat at the school. If more students have listed a school as their first preference than there are seats at the school, $66 \%$ of the seats at the school (i.e., two seats at schools $A$ and $B$, respectively, and four seats at school $C$ ) will be given to the students with the highest ranks. The remaining 
$33 \%$ (i.e., one seat at schools $A$ and $B$, respectively, and two seats at school $C$ ) will be assigned through a lottery. The lottery gives everyone participating the same chance and is therefore entirely independent of the ranks. For the students who were assigned a seat, the allocation procedure ends.

\section{Round 2:}

- Each student who did not receive a seat in the first round applies to the school he listed as his second preference. If he only listed one school, the allocation procedure ends, and he will receive no seat.

- If fewer or exactly as many students as there are remaining seats at a school have listed that school as their second preference, all of them get a seat at that school. If more students have listed a school as their first preference than there are seats still available at that school, the seats will be given to the students with the highest ranks. For the students who were assigned a seat, the allocation procedure ends.

\section{Round 3:}

- Each student who did not receive a seat in the first or second round applies to the school he listed as his third preference. If he only named two preferences, the allocation procedure ends, and he will not receive a seat.

- In the last round, there are fewer or exactly as many students as there are seats still available at a school who have listed the school as their third preference. All of them receive a seat at that school and the allocation procedure ends.

The allocation procedure ends when either none of the students are rejected by a school or when all rejected applicants have already applied to all schools on their preference list. A participant who has not received a seat at a school at this point is left without a seat.

It is irrelevant for the payoff whether you receive a seat at a school through your rank or through the lottery. Your payoff is exclusively determined by the school you are assigned to.

\section{Important Information for your Decision}

A computer executes the allocation procedure. The description above explains the procedure applied. After each round, you will be shown at which schools you and the other 11 participants have received a seat. Afterwards, the next round starts. The experiment ends after 24 rounds. 


\section{Example of the Allocation Procedure}

To illustrate the allocation procedure, we will now show you an example. In this example, there are 12 students and three schools (schools $A$ and $B$ have 3 seats to fill and school $C$ has 6 seats to fill). The students were assigned the ranks 1 to 12 . The student in rank 1 has the best grades, the student in rank 2 has the second-best grades etc. The following table shows the preference lists of all 12 participants.

\begin{tabular}{|c|c|c|c|}
\hline Student & First Preference & Second Preference & Third Preference \\
\hline Student in rank 1 & $A$ & $B$ & $C$ \\
\hline Student in rank 2 & $A$ & $B$ & $C$ \\
\hline Student in rank 3 & $B$ & $A$ & $C$ \\
\hline Student in rank 4 & $A$ & $B$ & $C$ \\
\hline Student in rank 5 & $C$ & $A$ & $C$ \\
\hline Student in rank 6 & $A$ & $B$ & $C$ \\
\hline Student in rank 7 & $C$ & $B$ & $A$ \\
\hline Student in rank 8 & $B$ & $A$ & $C$ \\
\hline Student in rank 9 & $C$ & $A$ & $B$ \\
\hline Student in rank 10 & $A$ & $B$ & $C$ \\
\hline Student in rank 11 & $A$ & $B$ & $C$ \\
\hline Student in rank 12 & $A$ & $C$ & $B$ \\
\hline
\end{tabular}

Notes: Preference lists of the students (example).

Important: The preference lists provided here are merely for illustrative purposes and are unrelated to your own decision situation.

We will now go through each round of the allocation procedure to illustrate how the procedure works.

\section{Round 1:}

- With their first preferences, the students with ranks 1, 2, 4, 6, 10, 11, and 12 apply to school $A$ which only offers three seats. The students with the two highest ranks (rank 1 and 2) are admitted to school $A$. One additional student (one of those ranked $4,6,10,11$, or 12 ) is admitted to school $A$ through the lottery. Let's say that this is the student in rank 11. Overall, three students will be admitted (rank 1 and 2 for the first two seats and rank 11 for the third seat because of the lottery). 
The other students in the ranks 4, 6, 10, and 12 do not receive a seat in the first round.

- Given their first preference, the students in ranks 3 and 8 apply to school $B$ which also has three seats to fill. Since there are fewer applicants than seats at the school, the students ranked 3 and 8 are admitted to school $B$.

- Given their first preferences, the students ranked 5th, 7th, and 9th apply to school $C$ which has six seats to fill. Since there are fewer applicants than seats at the school, the students ranked 5th, 7th, and 9th are admitted to school $C$.

- The allocation procedure ends for the students in ranks $1,2,3,5,7,8,9$, and 11 who have all received a seat. The students in ranks 4, 6, 10, and 12 did not receive a seat this round and go on to the second round.

\section{Round 2:}

- The students in ranks 4, 6, 10, and 12 have not yet received a seat. They apply to their second-most preferred school. Given their second preference, the students in ranks 4,6 , and 10 apply to school $B$ which still has one seat available. The student with the highest rank (rank 4 ) is admitted to school $B$. The students ranked 6th and 10 th do not receive a seat at school $B$.

- Given his second preference, the student ranked 12th applies to school $C$ which has three available seats left, and is admitted.

- The allocation procedure ends for the students in ranks 4 and 12 who have each received a seat. The students in ranks 6 and 10 have not received a seat yet and go into the third round.

\section{Round 3:}

- The students ranked 6th and 10th apply to their third-most preferred school, school $C$ and are admitted because school $C$ still has exactly two available seats. The allocation procedure ends.

The following assignments result from the allocation procedure:

\begin{tabular}{c||c|c|c|c|c|c|c|c|c|c|c|c} 
Rank & 1 & 2 & 3 & 4 & 5 & 6 & 7 & 8 & 9 & 10 & 11 & 12 \\
\hline \hline School & $A$ & $A$ & $B$ & $B$ & $C$ & $C$ & $C$ & $B$ & $C$ & $C$ & $A$ & $C$
\end{tabular}


Just to remind you again: the situation described above only illustrates the allocation procedure.

Do you have any questions?

Thank you for participating in the experiment!

\section{Quiz for Treatment IA33}

Please answer the following questions and raise your hand when you are done! One of the experimenters will then come to you.

There are six students and two schools: $A$ and $B$. School $A$ and $B$ each have three seats to fill. The students are ranked by their grades, the best student being in rank 1 and the worst student in rank 6 .

\begin{tabular}{l||c|c} 
& First Preference & Second Preference \\
\hline \hline Student in rank 1 & $A$ & $B$ \\
Student in rank 2 & $A$ & $B$ \\
Student in rank 3 & $B$ & $A$ \\
Student in rank 4 & $B$ & $A$ \\
Student in rank 5 & $B$ & $A$ \\
Student in rank 6 & $B$ & $A$ \\
\hline
\end{tabular}

Notes: The lottery for school $B$ draws the student in rank 6 . At which school is the student in rank 4 admitted? ( $A$ or $B$ )

\begin{tabular}{l||c|c} 
& First Preference & Second Preference \\
\hline \hline Student in rank 1 & $A$ & $B$ \\
Student in rank 2 & $A$ & $B$ \\
Student in rank 3 & $A$ & $B$ \\
Student in rank 4 & $A$ & $B$ \\
Student in rank 5 & $B$ & $A$ \\
Student in rank 6 & $B$ & $A$ \\
\hline
\end{tabular}

Notes: The lottery for school $A$ draws the student in rank 3. At which school is the student in rank 2 admitted? ( $A$ or $B$ ) 


\begin{tabular}{l||c|c} 
& First Preference & Second Preference \\
\hline \hline Student in rank 1 & $A$ & $B$ \\
Student in rank 2 & $A$ & $B$ \\
Student in rank 3 & $B$ & $A$ \\
Student in rank 4 & $A$ & $B$ \\
Student in rank 5 & $A$ & $B$ \\
Student in rank 6 & $B$ & $A$ \\
\hline
\end{tabular}

Notes: The lottery for school $A$ draws the student in rank 5 . At which school is the student in rank 5 admitted? ( $A$ or $B$ )

\begin{tabular}{l||c|c} 
& First Preference & Second Preference \\
\hline \hline Student in rank 1 & $A$ & $B$ \\
Student in rank 2 & $A$ & $B$ \\
Student in rank 3 & $A$ & $B$ \\
Student in rank 4 & $A$ & $B$ \\
Student in rank 5 & $A$ & $B$ \\
Student in rank 6 & $A$ & $B$ \\
\hline
\end{tabular}

Notes: The lottery for school $A$ draws the student in rank 4 . At which school is the student in rank 3 admitted? ( $A$ or $B$ )

\begin{tabular}{l||c|c} 
& First Preference & Second Preference \\
\hline \hline Student in rank 1 & $A$ & $B$ \\
Student in rank 2 & $A$ & $B$ \\
Student in rank 3 & $B$ & $A$ \\
Student in rank 4 & $B$ & $A$ \\
Student in rank 5 & $A$ & $B$ \\
Student in rank 6 & $A$ & $B$ \\
\hline
\end{tabular}

Notes: The lottery for school $A$ draws the student in rank 6 . At which school is the student in rank 1 admitted? ( $A$ or $B$ ) 


\section{Instructions for Treatment DA33 (translated from German)}

[See instructions for IA33 up to:]

\section{The Allocation Procedure}

In each round, you and the other students in your group will be randomly assigned a rank. The rank varies between 1 and 12, with each of the students occupying exactly one rank. Rank 1 means that the student has the best grades, rank 12 means that the student has the worst grades. Schools prefer students with better grades over students with worse grades.

In addition, a second list is created in which each student randomly receives a lottery number between 1 and 12. Each lottery number is assigned exactly once. The lottery numbers are determined anew in each round and are independent of the grades.

The allocation of the participants to the school seats works as follows: Two thirds of the seats are reserved for the students with the best grades. One third of the seats are allocated based on lottery numbers. Applicants with a lower lottery number have priority over applicants with a higher lottery number. Admission by means of grades and lottery numbers takes place in several rounds. In the first round, each student applies to the school that he listed as his first preference. If a school rejects the application of a student, he applies in later rounds to the school he listed as his second preference. If he is also rejected there in a later round, he applies to the school he listed as his third preference. The allocation of seats is temporary in each round. This means that a temporary seat in a later round can be awarded to a better-placed applicant.

Each round of the procedure consists of two parts: in Part 1, two thirds of the seats are awarded to the candidates with the highest ranks. There are two places at schools $A$ and $B$, and 4 places at school $C$. In Part 2, the remaining third of the seats (one seat at schools $A$ and $B$ and two seats at school $C$ ) are allocated based on the lottery numbers.

\section{Round 1:}

- Each student applies to the school he listed as his first preference. Schools $A$ and $B$ temporarily assign

- two seats to the two applicants with the best grades

- one seat among the remaining applicants with the lowest lottery number.

School $C$ temporarily assigns

- four seats to the four applicants with the best grades 
- two seats among the remaining applicants with the two lowest lottery numbers.

- Applicants who do not get a seat are finally rejected at the respective school. If there are not enough applicants at a school, the surplus seats remain temporarily free.

\section{Round 2:}

- Each student who was finally rejected at his most preferred school applies to the school he listed as his second preference. Each school compares the new applicants with the applicants from the previous round, which they have temporarily accepted.

- Schools $A$ and $B$ temporarily assign

- two seats to the two applicants with the best grades

- one seat among the remaining applicants with the lowest lottery number.

School $C$ temporarily assigns

- four seats to the four applicants with the best grades

- two seats among the remaining applicants with the two lowest lottery numbers.

- Applicants who do not get a seat are finally rejected at the respective school. If there are not enough applicants at a school, the surplus seats remain temporarily free.

\section{$(\ldots)$}

The procedure continues according to these rules. The procedure ends when a round is reached in which either one of the participants is no longer finally rejected by a school or each of the finally rejected candidates has applied to all the schools indicated on his preference list. At the end of the procedure, all temporary admissions become final: each student is given a seat at that school which has temporarily admitted him at that time. A student who was not admitted by any school now gets no seat.

In summary, the admission procedure works as follows:

1. In each round, the seats at the schools are only temporarily given. For example, a student who is rejected in a round by his preferred school will still have a chance to get a place at his second-most favourite school further in the course of the procedure, even if the latter has already assigned all its seats temporarily. 
2. All students apply twice if required: Once in Part 1 for one of the seats reserved for the students with the best grades and, if this application was not successful, once again for one of the seats in Part 2 awarded according to the lottery numbers.

3. A school which has finally rejected a student does not take him into account for the further course of the procedure. So, it does not help the student if he mentions the same school twice on his preference list. If a student is rejected by all the schools mentioned in his preference list, he will leave the procedure and will not be given any seat.

\section{Important Information for your Decision}

- If more than one participant applies to a school, in Part 1 of a round only the school grades determine which candidate is temporarily admitted. In Part 2 of a round, only the lottery numbers are used. For your payoffs, it is irrelevant whether you have received a seat at the school due to your grade or due to your lottery number.

- A computer executes the allocation procedure. The description above explains the procedure applied. After each round, you will be shown at which schools you and the other 11 participants have received a seat. Afterwards, the next round starts. The experiment ends after 24 rounds.

\section{Example of the Allocation Procedure}

To illustrate the allocation procedure, we will now show you an example. In this example, there are 12 students and three schools (schools $A$ and $B$ have 3 seats to fill and school $C$ has 6 seats to fill). The students are assigned the ranks 1 to 12 . The student in rank 1 has the best grades, the student in rank 2 has the second-best grades, etc. The following table shows the preference lists of all 12 participants. In addition, the last column of the table shows the lottery number of each student, assigned by a single lottery.

Important: The preference lists provided here are merely for illustrative purposes and are unrelated to your own decision situation.

We will now go through each round of the allocation procedure to illustrate how the procedure works.

\section{Round 1:}

\section{- Part 1:}

The students with ranks $1,2,4,6,10,11$, and 12 apply to school $A$, which offers two seats based on the ranks. The students at the two highest ranks (ranks 1 and 


\begin{tabular}{c||c|c|c||c} 
Student & $\begin{array}{c}\text { First } \\
\text { Preference }\end{array}$ & $\begin{array}{c}\text { Second } \\
\text { Preference }\end{array}$ & $\begin{array}{c}\text { Third } \\
\text { Preference }\end{array}$ & $\begin{array}{c}\text { Lottery } \\
\text { Number }\end{array}$ \\
\hline \hline Student in rank 1 & $A$ & $B$ & $C$ & 7 \\
Student in rank 2 & $A$ & $B$ & $C$ & 3 \\
Student in rank 3 & $B$ & $A$ & $C$ & 1 \\
Student in rank 4 & $A$ & $B$ & $C$ & 8 \\
Student in rank 5 & $C$ & $A$ & $C$ & 12 \\
Student in rank 6 & $A$ & $B$ & $C$ & 11 \\
Student in rank 7 & $C$ & $B$ & $A$ & 1 \\
Student in rank 8 & $B$ & $A$ & $C$ & 2 \\
Student in rank 9 & $C$ & $A$ & $B$ & 6 \\
Student in rank 10 & $A$ & $B$ & $C$ & 9 \\
Student in rank 11 & $A$ & $B$ & $C$ & 4 \\
Student in rank 12 & $A$ & $C$ & $B$ & 5 \\
\hline
\end{tabular}

Notes: Preference lists of the students and lottery numbers (example).

2) are temporarily admitted to school $A$. The students with the ranks 3 and 8 apply at their most preferred school $B$, which also awards two seats according to ranks. Since there are fewer applicants than seats available, the students with ranks 3 and 8 are temporarily admitted by school $B$. The students with ranks 5,7 , and 9 apply to school $C$, which awards four seats according to ranks. Since there are fewer applicants than seats available, the students with ranks 5, 7, and 9 are temporarily admitted by school $C$. 


\section{- Part 2:}

School $A$ assigns a further seat among the remaining applicants (ranks 4, 6, 10, 11, and 12) based on the lottery number. This seat goes to the student with rank 11 who has the lowest lottery number of the remaining candidates (4). A total of three students are admitted to school $A$ (ranks 1 and 2 for the first two seats and rank 11 through the lottery for the third seat). The students with ranks 4, 6, 10, and 12 do not get a seat in this round and are rejected by school $A$. At schools $B$ and $C$, no seats are allocated through the lottery, since there were enough seats for all applicants.

\section{Round 2:}

The students with ranks 4, 6, 10, and 12 have not yet received a seat. They apply to their second-most preferred school.

\section{- Part 1:}

The students with ranks 4,6 , and 10 apply to their second choice, school $B$. The student with rank 4 is temporarily admitted by school $B$, thus replacing the student with rank 8, who temporarily has no seat at school $B$. The students with ranks 6 and 10 do not receive a seat at school $B$. The student with rank 12 applies to his second choice, school $C$, which still has two available seats awarded according to the grades, and he is temporarily admitted.

\section{- Part 2:}

The students with ranks 6, 8, and 10 have not received any seat in Part 1 . They compete for the lottery seat at school $B$. Student 8 receives this seat because he has drawn the lowest lottery number (2).

\section{Round 3:}

\section{- Part 1:}

The students with ranks 6 and 10 do not have any seat after the second round and apply to their third school, school $C$. They are admitted, since school $C$ still has two available seats left. The allocation procedure ends.

The following assignments result from the allocation procedure:

\begin{tabular}{l||c|c|c|c|c|c|c|c|c|c|c|c} 
Rank & 1 & 2 & 3 & 4 & 5 & 6 & 7 & 8 & 9 & 10 & 11 & 12 \\
\hline \hline School & $A$ & $A$ & $B$ & $B$ & $C$ & $C$ & $C$ & $B$ & $C$ & $C$ & $A$ & $C$
\end{tabular}


Just to remind you again: the situation described above only illustrates the allocation procedure.

Do you have any questions?

Thank you for participating in the experiment!

\section{D.3 Quiz for Treatment DA33}

The quiz was the same as for IA33, the only difference being that the matching outcome had to be determined under DA33 instead of IA33.

\section{D.4 Screenshots}

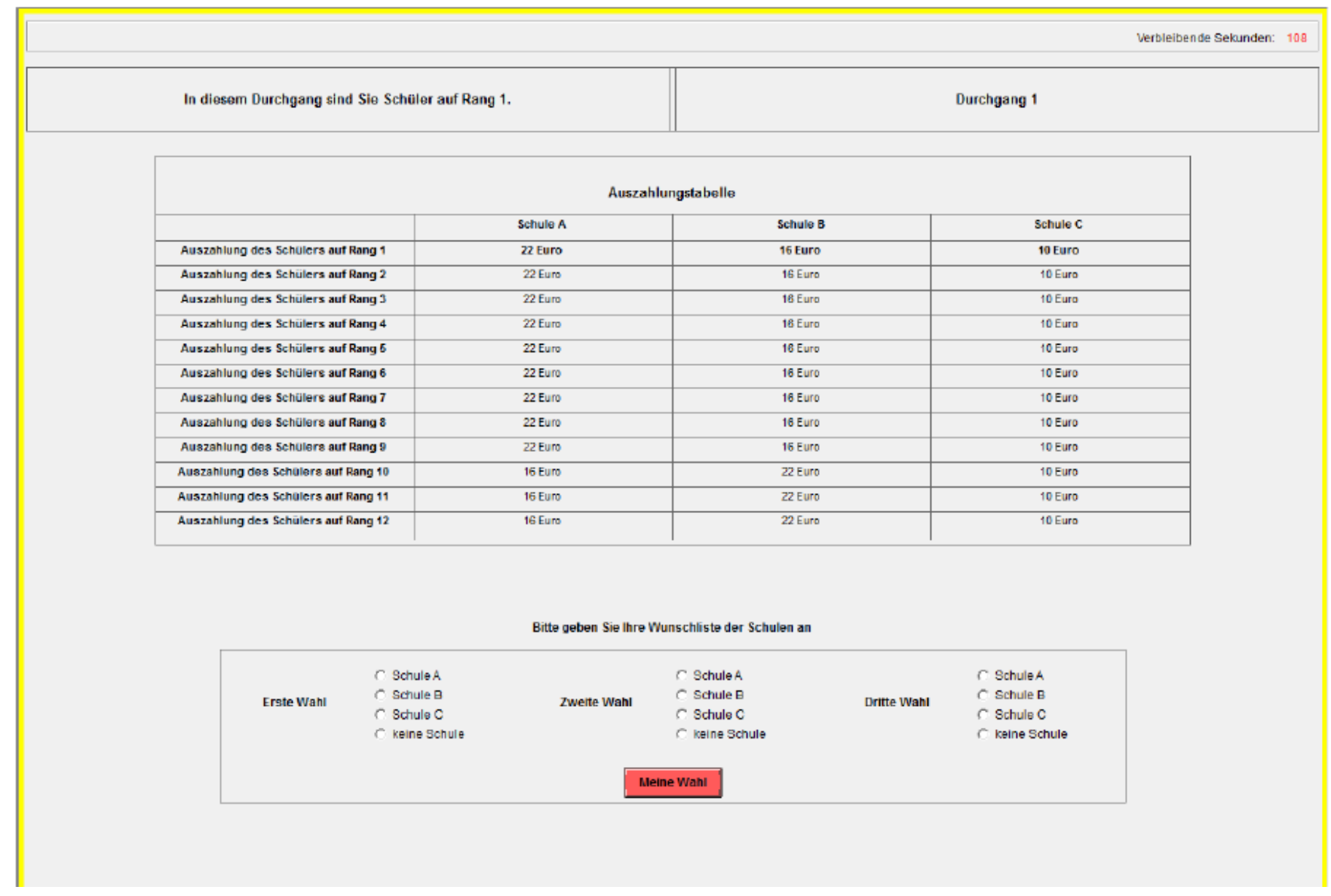




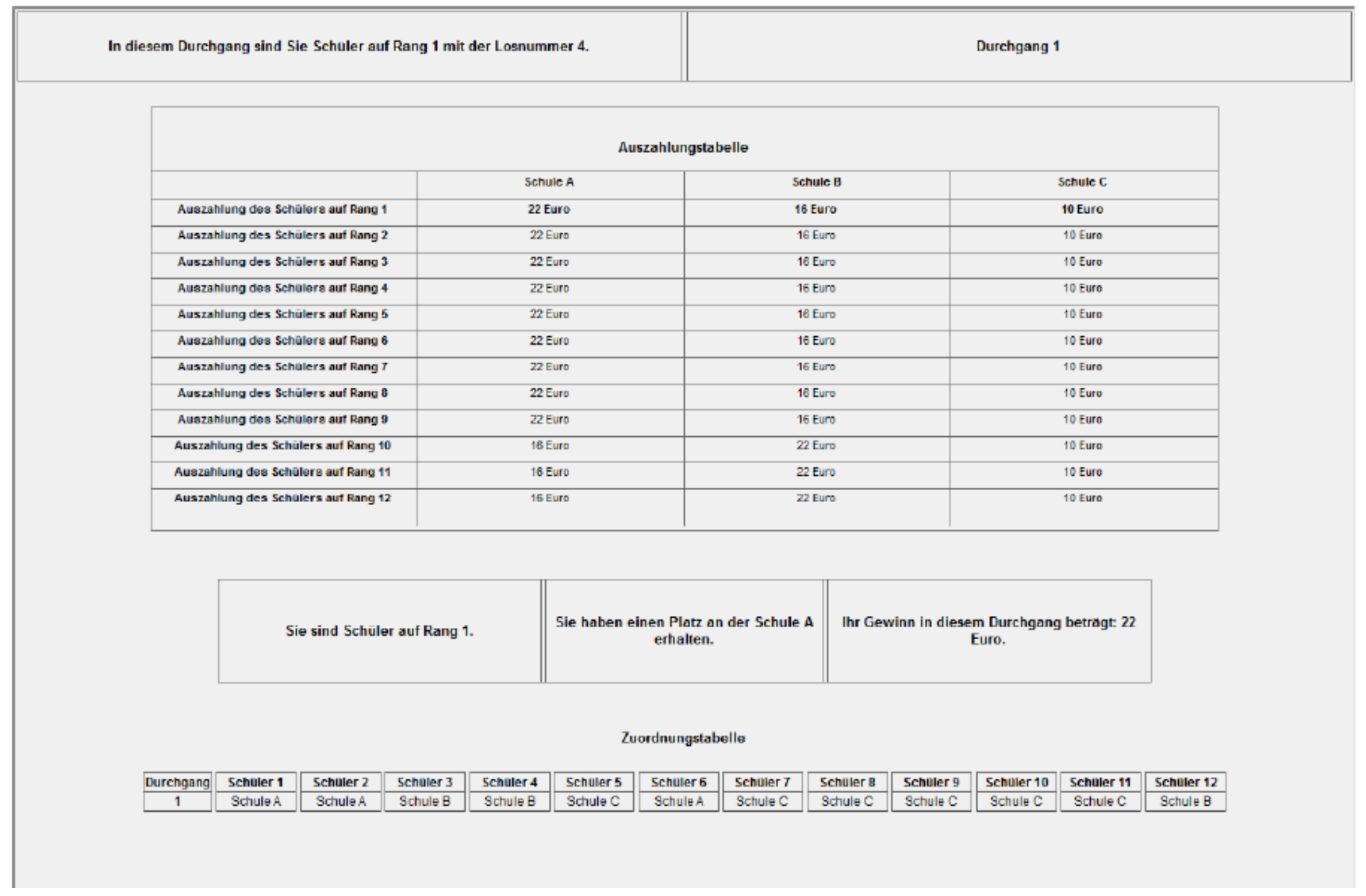




\section{References}

Abdulkadiroğlu, A., Y.-K. Che, and Y. Yasuda (2011). Resolving conflicting preferences in school choice: The "Boston mechanism" reconsidered. American Economic Review 101, 1-14.

Abdulkadiroğlu, A., P. A. Pathak, and A. E. Roth (2005). The New York City high school match. American Economic Review, Papers and Proceedings 95(2), 364-367.

Abdulkadiroğlu, A., P. A. Pathak, and A. E. Roth (2009). Strategy-proofness versus efficiency in matching with indifferences: Redesigning the NYC high school match. American Economic Review 99(5), 1954-1978.

Abdulkadiroğlu, A., P. A. Pathak, A. E. Roth, and T. Sönmez (2005). The Boston public school match. American Economic Review, Papers and Proceedings 95(2), 368-371.

Abdulkadiroğlu, A., P. A. Pathak, A. E. Roth, and T. Sönmez (2006). Changing the Boston school-choice mechanism: Strategy-proofness as equal access. NBER Working Paper No. 11965.

Abdulkadiroğlu, A. and T. Sönmez (2003). School choice: A mechanism design approach. American Economic Review 93, 729-747.

Allen, R., S. Burgess, and L. McKenna (2014). School performance and parental choice of school: secondary data analysis. Research Report DFERR310, https://www.gov.uk/government/uploads/system/uploads/attachment_data/ file/275938/RR310_-_School_performance_and_parental_choice_of_school.pdf.

Basteck, C., K. Huesmann, and H. Nax (2015). Matching practices for secondary schools Germany. MiP Country Profile 21, http://www .matching-in-practice.eu/wp-content/ uploads/2015/01/MiP_-Profile_No.21.pdf.

Basteck, C. and M. Mantovani (2018). Cognitive ability and games of school choice. Games and Economic Behavior 109, 156-183.

Bó, I. and R. Hakimov (2018). Iterative versus standard deferred acceptance: Experimental evidence. SSRN Working Paper, http://dx.doi.org/10.2139/ssrn.2860738.

Bohlmark, A., H. Holmlund, and M. Lindahl (2016). Parental choice, neighbourhood segregation or cream skimming? an analysis of school segregation after a generalized choice reform. Journal of Population Economics 29, 1155-1190.

Calsamiglia, C. and M. Güell (2018). Priorities in school choice: The case of the Boston mechanism in Barcelona. Journal of Public Economics 163, 20-36.

Calsamiglia, C., F. Martínez-Mora, and A. Miralles (2017). Sorting in public school districts under the Boston mechanism. Working Paper, http://humcap.uchicago.edu/RePEc/hka/ wpaper/Calsamiglia_Martinez-Mora_etal_2017_sorting-boston-mechanism.pdf. 
Chen, L. and J. S. Pereyra (2018). Self-selection in school choice. Working Paper, http: //dx.doi.org/10.2139/ssrn.2880182.

Chen, Y. and T. Sönmez (2006). School choice: An experimental study. Journal of Economic Theory 127, 202-231.

Coldron, J., E. Tanner, S. Finch, L. Shipton, C. Wolstenholme, B. Willis, S. Demack, and B. Stiell (2008). Secondary school admissions. Project Report, London, Department for Children, Schools and Families. Available from Sheffield Hallam University Research Archive (SHURA) at http://shura.shu.ac.uk/183/.

Ding, T. and A. Schotter (2017). Matching and chatting: An experimental study of the impact of network communication on school- matching mechanisms. Games and Economic Behavior 103, $94-115$.

Doğan, B. and B. Klaus (2018). Object allocation via immediate-acceptance: Characterizations and an affirmative action application. Journal of Mathematical Economics 79, 140-156.

Dur, U., S. D. Kominers, P. A. Pathak, and T. Sönmez (2018). Reserve design: Unintended consequences and the demise of boston's walk zones. Journal of Political Economy 126(6), $2457-2479$.

Echenique, F., L. Yariv, and A. Wilson (2016). Clearinghouses for two-sided matching: An experimental study. Quantitative Economics 7(2), 2679-2694.

Ehlers, L. and B. Klaus (2016). Object allocation via deferred-acceptance: Strategy-proofness and comparative statics. Games and Economic Behavior 97, 128-146.

Ehlers, L. and J. Massó (2015). Matching markets under (in)complete information. Journal of Economic Theory 157, 295-317.

Epple, D. and R. Romano (2008). Educational vouchers and cream skimming. International Economic Review 49(4), 295-317.

Epple, D. and R. E. Romano (1998). Competition between private and public schools, vouchers, and peer-group effects. American Economic Review 88(1), 33-62.

Ergin, H. and T. Sönmez (2006). Games of school choice under the Boston mechanism. Journal of Public Economics 90, 215-237.

Featherstone, C. and M. Niederle (2016). Improving on strategy-proof school choice mechanisms: An experimental investigation. Games and Economic Behavior 100, 353-375.

Fischbacher, U. (2007). z-tree: Zurich toolbox for ready-made economic experiments. Experimental Economics 10(2), 171-178. 
Gale, D. and L. S. Shapley (1962). College admissions and the stability of marriage. American Mathematical Monthly 69, 9-15.

Hakimov, R. and D. Kübler (2019). Experiments in matching markets: A survey. Working Paper, https://rationality-and-competition.de/wp-content/uploads/ discussion_paper/153.pdf.

Hassidim, A., A. Romm, and R. I. Shorrer (2016). "Strategic" behavior in a strategy-proof environment. EC'16, http://dx.doi.org/10.2139/ssrn.2784659.

Hatfield, J. W., F. Kojima, and Y. Narita (2016). Improving schools through school choice: A market design approach. Journal of Economic Theory 166, 186-211.

Klijn, F., J. Pais, and M. Vorsatz (2013). Preference intensities and risk aversion in school choice: A laboratory experiment. Experimental Economics 16(1), 1-22.

Miralles, A. (2009). School choice: The case for the Boston mechanism. Working Paper.

Nathanson, L., S. Corcoran, and C. Baker-Smith (2013). High school choice in New York City: A report on the school choices and placements of low-achieving students. Institute of Education Sciences Report, https://files.eric.ed.gov/fulltext/ED541824.pdf.

Noden, P., A. West, and A. Hind (2014). Banding and ballots: Secondary school admissions in England. The Sutton Trust, London, UK, https://www.suttontrust.com/wp-content/ uploads/2014/02/banding-and-ballots-report.pdf.

Pais, J. and A. Pinter (2008). School choice and information: An experimental study on matching mechanisms. Games and Economic Behavior 64, 303-328.

Pathak, P. and T. Sönmez (2013). School admissions reform in Chicago and England: Comparing mechanisms by their vulnerability to manipulation. American Economic Review 103, 80-106.

Pathak, P. A. and T. Sönmez (2008). Leveling the playing field: Sincere and sophisticated players in the Boston mechanism. American Economic Review 98(4), 1636-1652.

Ruijs, N. and H. Oosterbeek (2019). School choice in Amsterdam: Which schools are chosen when school choice is free? Education Finance and Policy 14(1), 1-30.

Solakova, B. (2011). The direct acceptance mechanism with a lottery: How does the lottery element affect truth revelation and efficiency in the school choice problem? Master's thesis, University of Lausanne, Switzerland.

Westkamp, A. (2013). An analysis of the German university admissions system. Economic Theory 53, 561-589. 